\title{
An efficient CDEM-based method to calculate full-scale fragment field of warhead
}

\author{
Haozhe Wang ${ }^{\mathrm{a}}$, Chunhua Bai ${ }^{\mathrm{a}}$, Chun Feng ${ }^{\mathrm{b}, \mathrm{c}, *}$, Kun Xue ${ }^{\mathrm{a}}$, Xinguang Zhu ${ }^{\mathrm{b}, \mathrm{c}}$ \\ ${ }^{a}$ State Key Laboratory of Explosive Science, Beijing University of Technology, Beijing 10000, China \\ ${ }^{\mathrm{b}}$ Institute of Mechanics, Chinese Academy of Sciences, Beijing, China \\ ${ }^{\mathrm{c}}$ University of Chinese Academy of Sciences (UCAS), School of Engineering Science, Beijing, China
}

\section{A R T I C L E I N F O}

\section{Keywords:}

Fragments field

Continuum-based discrete element method

Cross-scale calculation

Detonation product leakage algorithm

\begin{abstract}
A B S T R A C T
Warhead's full-scale fragment field has great significance to military production and storage safety. In this paper, an improved Continuum-Discontinuum Element Method (CDEM) will be used for the first time to solve the problems of large deformation and cross-scale calculations for warheads, describing the complete process from casing breakage to fragment scattering and landing. A fragment equivalent layer-detonation product escape algorithm is further introduced to increase calculation efficiency, and the fragmentation field is post-processed using a penetrable virtual target board algorithm. This method simulates warhead fragmentation in the full space-time domain, laying a foundation for subsequent study of questions like warhead fragmentation safety distance and fuze delay arming time. Comparison with warhead static explosion and ballistic gun testing data verify the effectiveness of this method.
\end{abstract}

\section{Introduction}

Research on warhead fragmentation in full-scale has direct significance for warhead effectiveness and corresponding production and storage safety. Due to the large number and high density of warhead fragments, however, as well as the high number of interfering factors like flames, dust, impact, vibrations, and ineffective debris, it is extremely difficult to test and measure all fragment state parameters by experiment [1].

Simulation research can roughly divide the research area into shortand long-range fragmentation fields based on distance to the explosion center.

The short-range fragmentation field is usually that within the intensive kill radius. After the warhead is detonated, the fragments are accelerated by the detonation products within this area to their maximum speed [2]. Initial fragment velocity is high, flight time is short, and the effects of gravity and resistance are not significant.

This portion of the research is usually on the basis of destruction, often using the finite element method to describe the interaction between the explosive detonation products and the casing. For example, Marinko et al. [3]., applied the AUTODYN program to numerically simulate the detonation-driven process of a prefabricated fragmentation warhead. Gold et al. [4,5]. used a static field explosion test and the ALE numerical simulation method to study the explosion of a prefabricated fragment warhead. Hopson [6] used the CTH software to calculate the explosion process of AerMet-shelled warhead. Prytz [7], Jian-Yu [8], Jonathan P [9], Rusinek [10], Zhang [11], Weiping [12] et al. also carried out similar research, obtaining data in good agreement with testing.

After detonation, the expansion of detonating gas lead to large deformation and cracking of shell. In order to accurately describe the fracture and breakage in casing materials, a lot of works have been done. Such as Khader et al. [13] presents a methodology for stochastic modeling of the fracture in different materials based on the theory of fracture energy. Furthermore, sensitivity analysis has been applied to identify the key input parameters influencing the energy conversion factor (ECF) of materials above [14].

Timon et al. [15] described an approach for modeling discrete cracks in meshfree methods, where the crack is modeled by splitting particles located on opposite sides of the associated crack segments. Rabczuk, and Belytschko, [16] presented a method by which the crack is modeled by a local enrichment of the test and trial functions with a sign function (a variant of the Heaviside step function), so that the discontinuities are along the direction of the crack.

Beside, Shafiei [17], Areias [18], Oliver [19], Gravouil [20], Wu and Nguyen[21], Y. Zhang [37-39] also proposed a variety of effective numerical methods to describe the fracture and breakage in materials. The long-range fragmentation field usually extends from the end of the

\footnotetext{
* Corresponding author.

E-mail address: fengchun@imech.ac.cn (C. Feng).
} 
intensive kill radius to the fragment landing distance. Ballistic flight in this area is mainly subject to air resistance and gravity. Research on this zone usually involves safety, such as warhead production, storage and transport, destruction safety, and fuze safety distance, etc.

Because of the large number of fragments, long flight distance, the computational efficiency of solid-fluid coupling in grid-based calculation is not satisfactory. Research on this zone mainly uses iterative calculations to describe the motion trajectory of fragments. The shotline technique developed by the Dutch TNO-Prins Maurits Laboratory (TNO-PML) [22], as an example, given initial conditions including the mass, density, and air resistance for a single fragment in flight, iteratively calculates the magnitude and direction of the resultant force upon it from gravity and air resistance.

Similarly, Moore et al. [23] . used static short-range explosion test data as initial conditions in shot-line calculations to find the dynamic safe fragmentation distance of a guided missile. Chrostowski, [24], developed a iterative calculation software named as HAZX for assessing explosive hazards when the Quantity-Distance (Q-D) safe separation requirements are violated. Gan [25] put forward a new method based on HAZX and Monte Carlo (MC) simulation to perform detailed, sitespecific debris hazard analysis. Jiang et al. [26] assigned initial parameters to the fragments using theories related to warhead fragmentation (the Gunny formula and Mott distribution, etc.), then simulated the trajectories of each fragment through shot lines method to obtain the full fragmentation field distribution.

The finite element and iterative calculation method have superiority and the insufficiency respectively in describing full fragment field. The former can accurately describe the processes of warhead casing disintegration and fragment dispersion driven by the detonation products, but due to the difficulty of cross-scale calculation, this method is not highly applicable to long-range calculations. Iterative calculation has been developed over many years and can accurately describe the longrange fragment field, but the fragments' initial motion conditions (velocity, direction, and rotation) still require external input. Current studies mainly use theoretical formulas or static explosion test dispersion data as inputs. The former is imprecise, while the latter is timeconsuming and costly, restricting the accuracy and applicability of iterative calculation.

Based on the Continuum-Discontinuum Element Method (CDEM) [27], this study combines the finite element method with particulate discrete element (iterative) modeling. The results of the former are transfered to the latter as initial conditions, describing the full process from casing disintegration to fragment dispersal and landing, quickly and accurately calculating the full warhead fragment field in the space and time domains.

\section{Algorithm description}

By combining finite and discrete elements, CDEM not only simulates the materials' elastoplastic deformation and contact impacts, but also their progressive failure process from continuous to discontinuous. CDEM has a wide range of applications in geology, rock-soil mechanics, blasting, and penetration [28].

In order to efficiently simulate the dispersion field of prefabricated fragments, this paper improves upon traditional CDEM by coupling finite and particulate discrete elements. The explosive and metal shell are described using Langrarian finite elements; the prefabricated fragments are described as individual particles arranged spatially, and the space where they are located is equivalent to a layer of a continuous medium, described as a finite element, as shown in Fig. 1.

At the start of the calculation, the explosive element and shell element are activated. Finite element calculation is performed on the equivalent prefabricated fragment elements. The explosion is ignited at a given position in the explosive, and the blasting gas drives the shell and equivalent prefabricated fragment layer expanding outside. Meanwhile, an equivalent detonation product escape algorithm is

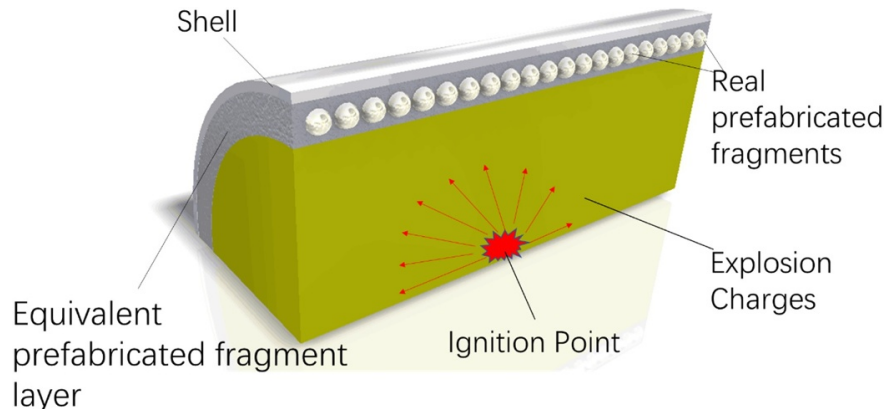

Fig. 1. Warhead discretization.

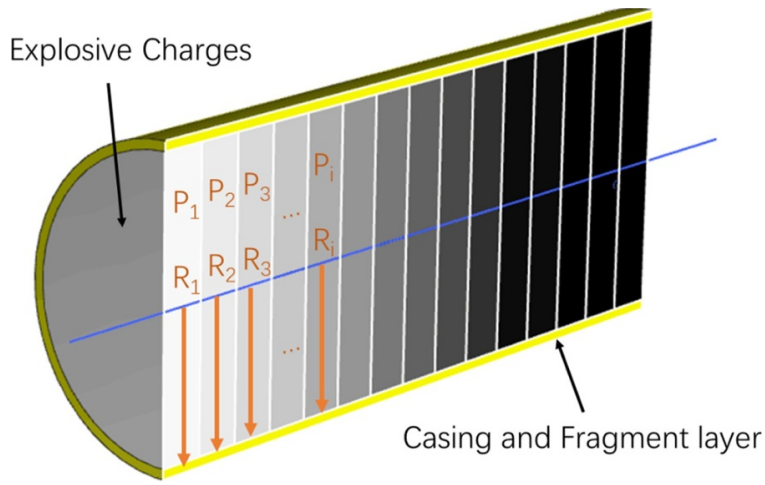

Fig. 2. Explosive gas escape algorithm.

introduced to obtain accurate gas pressure. After the velocity of the prefabricated fragment equivalent layer reaches constant stage, finite element calculation is stopped, and all finite elements are inactivated. At the same time, discrete element calculation is activated, and the initial velocity of the discrete elements is inherited from the corresponding finite element. After that, the flying process with drag force of the prefabricated fragment is simulated, and the dispersal field and target shooting is analyzed.

\subsection{Finite element solution}

\subsubsection{Basic principles}

For a general dynamics of elasto-plasticity, the basic equations and boundary conditions are shown in Eqs. (1) and (2).

$\left\{\begin{array}{ccc}\text { equilibriumequation } & \sigma_{i j, j}+f_{i}=\rho a_{i} & (i, j=x, y, z) \\ \text { geometricalequation } & \varepsilon_{i j}=\frac{1}{2}\left(u_{i, j}+u_{j, i}\right) & (i, j=x, y, z) \\ \text { constitutiveequation } & \sigma_{i j}=f\left(\varepsilon_{i j}, c_{1}, c_{2}, \ldots\right) & (i, j=x, y, z)\end{array}\right.$

$\left\{\begin{array}{cc}\sigma_{i j} n_{j}=T_{i} & \text { on } \mathrm{S}_{\sigma} \\ u_{i}=\bar{u}_{i} & \text { on } \mathrm{S}_{u}\end{array}\right.$

where $\varepsilon_{i j}, \sigma_{i j}$ are strain and stress tensor, fi is the volume force vector, $\rho$ is density, ai and ui are acceleration and displacement vector, c1, c2 are material parameters, $\mathrm{nj}$ is the outward unit vector at boundary face $\mathrm{S}_{\sigma}$, $T_{i}$ and $\bar{u}_{i}$ denote face force and displacement condition at boundary.

A real time updating FEM is adopted, and the incremental method is used to calculate the stress and node deformation force of the finite elements at Gauss point $\mathrm{i}$ :

$\left\{\begin{array}{l}\Delta \boldsymbol{\varepsilon}_{i}=\mathbf{B}_{i} \Delta \mathbf{u}_{e} \\ \boldsymbol{\varepsilon}_{i}=\sum \Delta \boldsymbol{\varepsilon}_{i} \\ \sigma_{i}=f\left(\boldsymbol{\varepsilon}_{i}, \Delta \boldsymbol{\varepsilon}_{i}, c_{1}, c_{2}, \cdots\right) \\ \mathbf{F}_{e}=\sum_{i=1}^{N} \mathbf{B}_{i}^{T} \sigma_{i} w_{i} J_{i}\end{array}\right.$

where $\mathbf{B}_{i}, \Delta \varepsilon_{i}, w_{i}$, and $J_{i}$ are respectively the strain matrix, incremental 


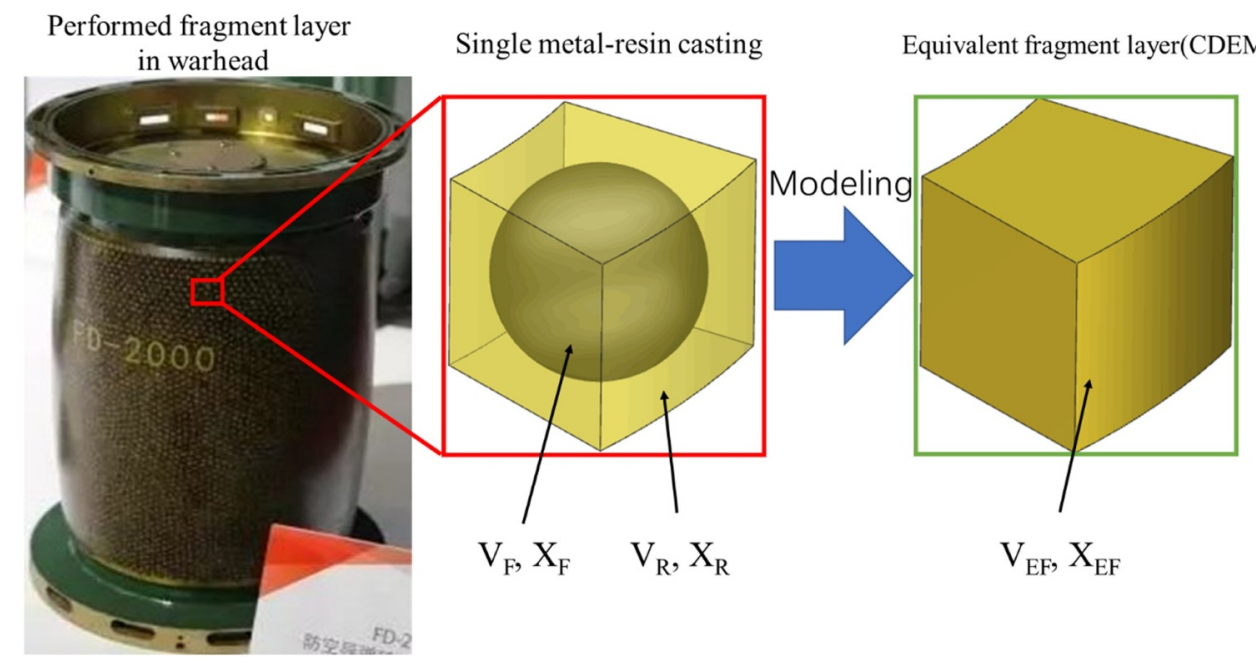

Fig 3. Diagram of fragment layer.

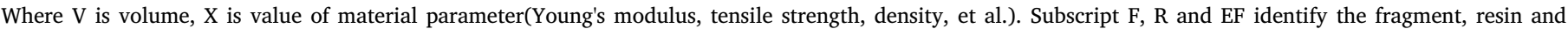
equivalent fragment layer.

Table 1

Determination of equivalent layer parameters.

\begin{tabular}{ll}
\hline Parameter & Determination of value \\
\hline Young's modulus & $X_{E F}=\left(V_{F} \cdot X_{F}\right) /\left(V_{F}+V_{R}\right)+\left(V_{R} \cdot X_{R}\right) /\left(V_{F}+V_{R}\right)$ \\
Tensile strength & $X_{E F}=\operatorname{Min}\left(X_{F}, X_{R}\right)$ \\
Density & $X_{E F}=\left(V_{F} \cdot X_{F}\right) /\left(V_{F}+V_{R}\right)+\left(V_{R} \cdot X_{R}\right) /\left(V_{F}+V_{R}\right)$ \\
Poisson ratio & $X_{E F}=\left(V_{F} \cdot X_{F}\right) /\left(V_{F}+V_{R}\right)+\left(V_{R} \cdot X_{R}\right) /\left(V_{F}+V_{R}\right)$
\end{tabular}

strain tensor, incremental stress tensor, integral coefficient, and Jacobian determinant at Gauss point $i ; \Delta \varepsilon_{i} \boldsymbol{\varepsilon}_{i}$ and $\boldsymbol{\sigma}_{i}$ are the incremental strain tensor, strain tensor and stress tensor at Gauss point $i ; \Delta \mathbf{u}_{e}$, and $\mathbf{F}_{e}$ are nodal displacement vector, and nodal deformation force vector; and $N$ is the number of Gauss points.

After calculating the deformation force of the node, we calculate its resultant force:

$\mathbf{F}=\mathbf{F}^{E}+\mathbf{F}^{e}+\mathbf{F}^{c}+\mathbf{F}^{d}$

where $\mathbf{F}$ is the nodal resultant force, $\mathbf{F}^{E}$ is its external force, $\mathbf{F}^{e}$ is its force contributed by deformation of the finite element, $\mathbf{F}^{c}$ is its force contributed through the contact interface, and $\mathbf{F}^{d}$ is the nodal damping force.

The node's motion is calculated using Euler's forward interpolation method:
$\begin{cases}\mathbf{a}=\mathbf{F} / m & \mathbf{v}=\sum_{t=0}^{T_{\text {now }}} \mathbf{a} \Delta t \\ \Delta \mathbf{u}=\mathbf{v} \Delta t & \mathbf{u}=\sum_{t=0}^{T_{\text {now }}} \Delta \mathbf{u}\end{cases}$

where $\mathbf{a}$ is the nodal acceleration vector, $\mathbf{v}$ is its velocity vector, $\Delta \mathbf{u}$ is its incremental displacement vector, $\mathbf{u}$ is its displacement vector, $m$ is its mass, and $\Delta t$ is the time step. An explicit solution process can be realized based on alternate computation of Eqs. (4) and (5).

When simulating large deformation problems, the distortion of element will happen. To solve the problem, incremental method should be adopted and strain matrix [B] should be used to calculate deformation force (Eq. (3)). Besides, strain matrix [B] should be renewed at each time step.

This method is verified by large rotation calculation examples in our former research [29].

\subsubsection{Explosive model}

This study uses the Landau firing model to describe the adiabatic constant detonation and gas detonation processes. The model uses the Landau-Stan Newkovic formula (rate equation) Eq. (6)) to calculate the gas expansion pressure, where $\gamma_{1}$ and $\gamma_{2}$ indicate the adiabatic indices in the first and second stages respectively (for general condensed explosives, $\gamma_{1}=3$ and $\left.\gamma_{2}=4 / 3\right) ; P(\mathrm{~Pa})$ and $V\left(\mathrm{~m}^{3}\right)$ respectively indicate the instantaneous pressure and volume of the high-pressure explosion frontier; $P_{0}$ and $V_{0}$ respectively indicate the pressure of the frontier in the initial period and the volume of the explosives; and $P_{k}$ and $V_{k}$ respectively indicate the pressure and volume at the high-pressure

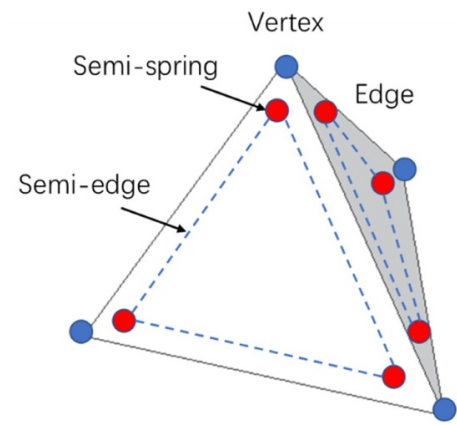

Three-dimensional tetrahedron

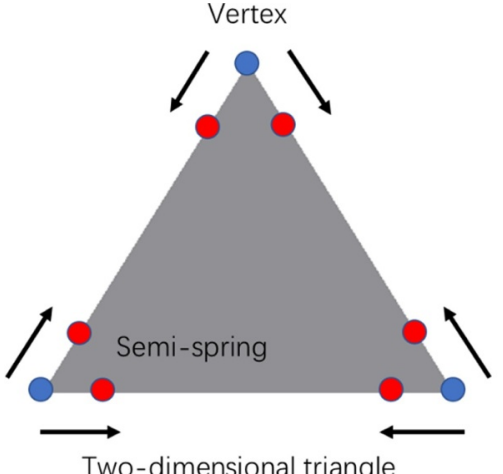

Fig. 4. Semi-spring and semi-edge diagram [29]. 


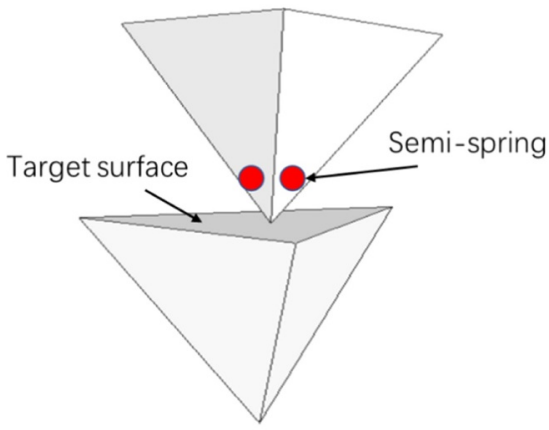

Semi-spring/target surface contact

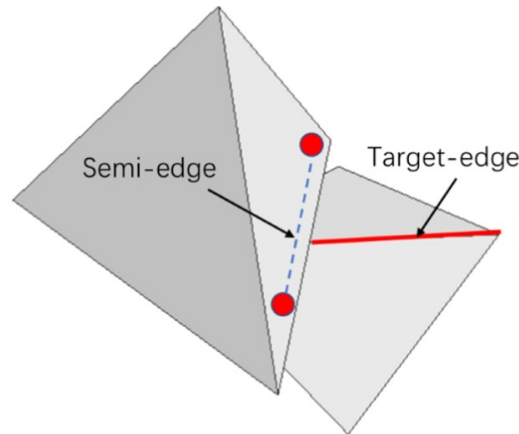

Semi-edge/target edge contact

Fig. 5. Two types of contact pairings [29].

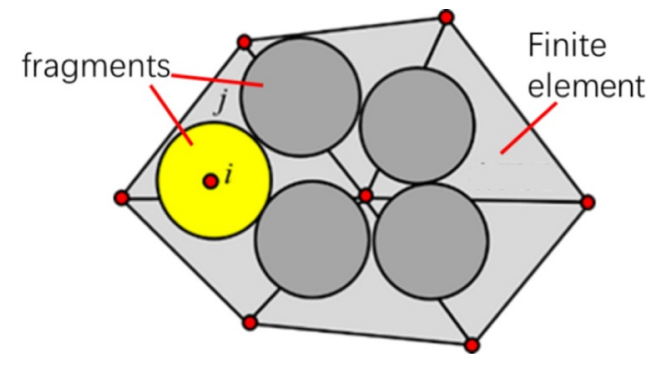

Fig. 6. Diagram of fragment velocity transformation.

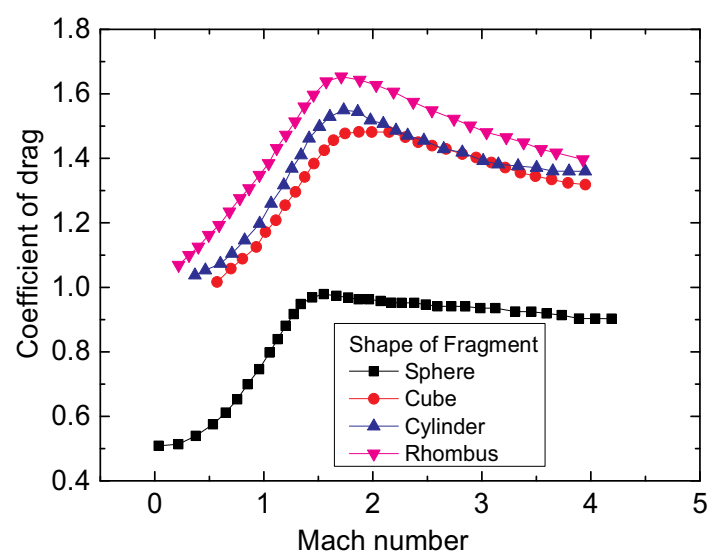

Fig 7. The correspondence between coefficient of drag and Mach number. frontier at the boundary of the two-stage adiabatic process. $P_{k}$ and $P_{k}$ are given by Eqs. (5) and ((6) respectively, where $Q_{w}$ is explosion heat $(\mathrm{J} / \mathrm{kg}), \rho_{w}$ is charge density $\left(\mathrm{kg} / \mathrm{m}^{3}\right)$, and $D$ is detonation velocity $(\mathrm{m} / \mathrm{s})$.

$\begin{cases}P V \gamma_{1}=P_{0} V_{0}^{\gamma_{1}} & P \geq P_{k} \\ P V \gamma^{\gamma_{2}}=P_{k} V_{k}^{\gamma_{2}} & P<P_{k}\end{cases}$

$P_{k}=P_{0}\left\{\frac{\gamma_{2}-1}{\gamma_{1}-\gamma_{2}}\left[\frac{\left(\gamma_{1}-1\right) Q_{w} \rho_{w}}{P_{0}}-1\right]\right\}^{\frac{\gamma_{1}}{\gamma_{1}-1}}$

$P_{0}=\frac{\rho_{w} D^{2}}{2\left(\gamma_{1}+1\right)}$

In addition, when the pressure produced by the explosion exceeds the Chapman-Jouguet (C-J) pressure $\left(P_{C J}\right)$, it is set to the CJ pressure:

if $P>P_{C J} \quad P=P_{C J}$

In the calculations, $V_{0}$ is the initial volume of the explosive element and $V$ is the current volume. Therefore, large deformation is required to update the element coordinates in real time and thereby calculate element volume.

The Landau model requires a detonation position and time, determining whether to calculate explosion pressure for a particular explosive element based on detonating speed $D$. Setting the firing time of an explosive (including some elements) as $t_{0}$, the coordinates of the firing point as $\left(x_{0}, y_{0}, z_{0}\right)$, the distance from the center of a given explosive element to the firing point as $d$, then the firing time of that element is $t_{1}=d / D+t_{0}$. Only when the firing time $t>t_{1}$ is the explosive pressure calculated for that element, according to Formula (10), in which $P_{r}$ is the real explosive pressure, and $f(P)$ is the detonation

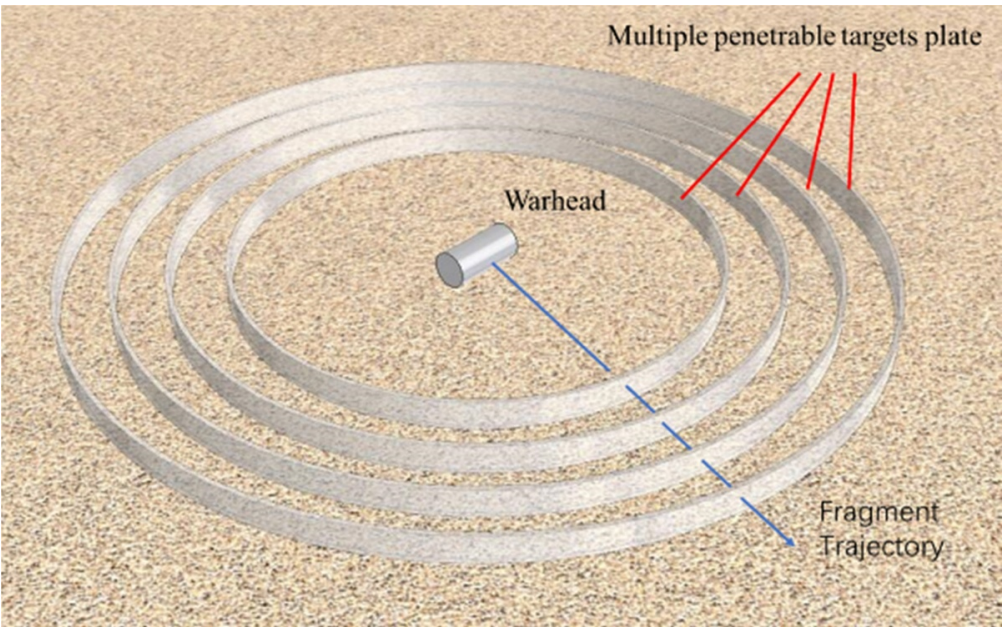

Fig. 8. Diagram of penetrable annular vertical target. 


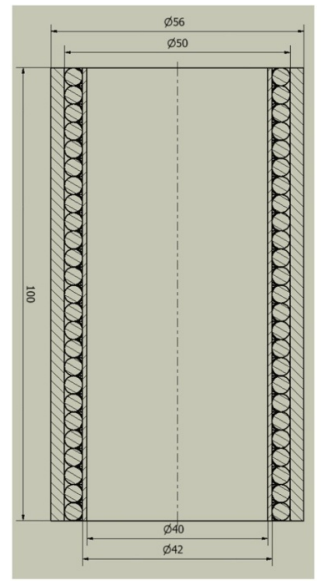

(a)

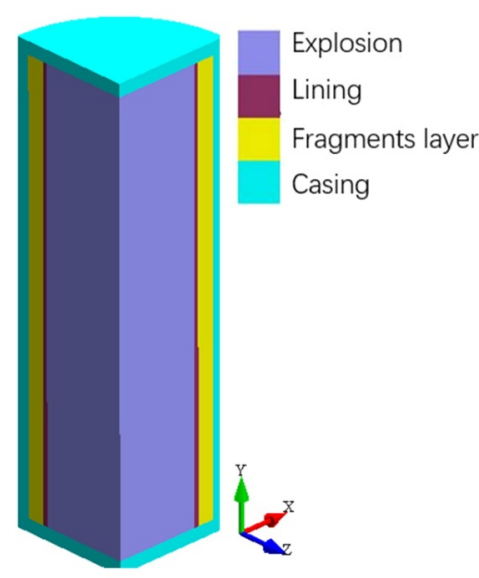

(b)
Fig 9. (a) Warhead diagram (b) CDEM finite element model.

product equation of state (obtained from Formula (4)). $\xi$ is the energy release rate, obtained from Formula (11), in which $V_{e}$ is the initial volume of the element and $A_{e-\max }$ is its maximum volume.

$P_{r}=\xi f(P)$

$\xi=\left\{\begin{array}{l}\min \left(\frac{2\left(t-t_{1}\right) D A_{e-\max }}{3 V_{e}}, 1\right) \text { if } t>t_{1} \\ 0 \quad \text { if } t \leq t_{1}\end{array}\right.$

When coupling with metal shell, the explosive element and shell element should be own common nodes, through which the explosive pressure generated by the explosive element automatically acts on the surrounding shell element.

In order to blasting pressure reduction caused by gas leakage due to fragmentation of shell, a detonation gas escape algorithm is further introduced.

As, shown in Fig 2, the explosive is divided into $\mathrm{N}$ segments along the axial direction, each of whose length is greater than or equal to the maximum element length. The radial displacement of each segment's outermost explosive node is calculated, and the current average radius $r_{i}$ is calculated as follows:

$r_{i}=r_{0}+\frac{1}{M} \sum_{k=1}^{M} u_{k}$

where $M$ is the total number of outermost unit nodes in the $i^{\text {th }}$ segment, $u_{k}$ is the outermost vertical displacement of the $k^{\text {th }}$ node, and $r_{0}$ is the initial radius of the explosive charge.

Next, the current radius of the explosive elements in each segment is used to calculate the pressure drop caused by loss of explosive gas in all elements of that segment, as follows:

$P_{n}=P_{r} \times \alpha\left(r_{c r} / r_{i}\right)^{\beta}$

where $r_{r c}$ is the critical radius, $P_{n}$ is the new pressure in a given explosive element, and $\alpha$ and $\beta$ are the pressure correction coefficient and index.

\subsubsection{Casing and equivalent layer model}

The metal shell and equivalent layer are described using a strain softening Tresca elasto-plasticity model with tensile cutoff. The strength criteria is shown in Formula (14), where $\sigma_{1}, \sigma_{2}$ and $\sigma_{3}$ are minimal, medium and maximal principal stress, $\tau_{c}$ is shear strength, $\sigma_{t}$ is tensile strength.

$\left\{\begin{array}{l}0.5\left(\sigma_{1}-\sigma_{3}\right) \geq \tau_{c} \\ \sigma_{3} \geq \sigma_{t}\end{array}\right.$

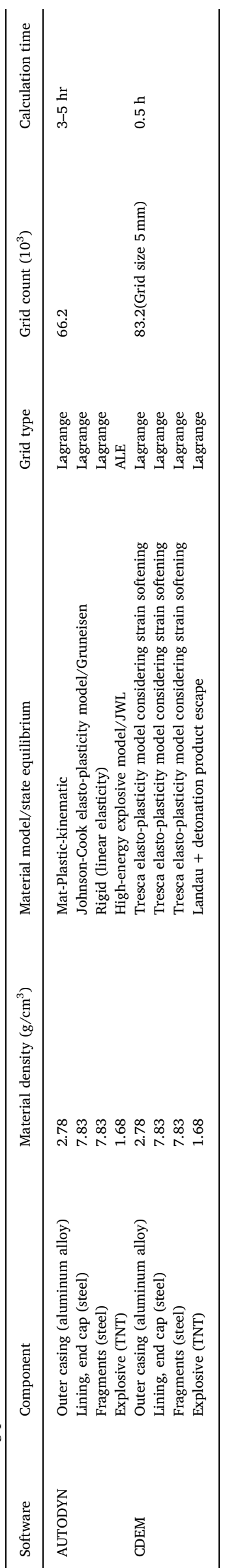




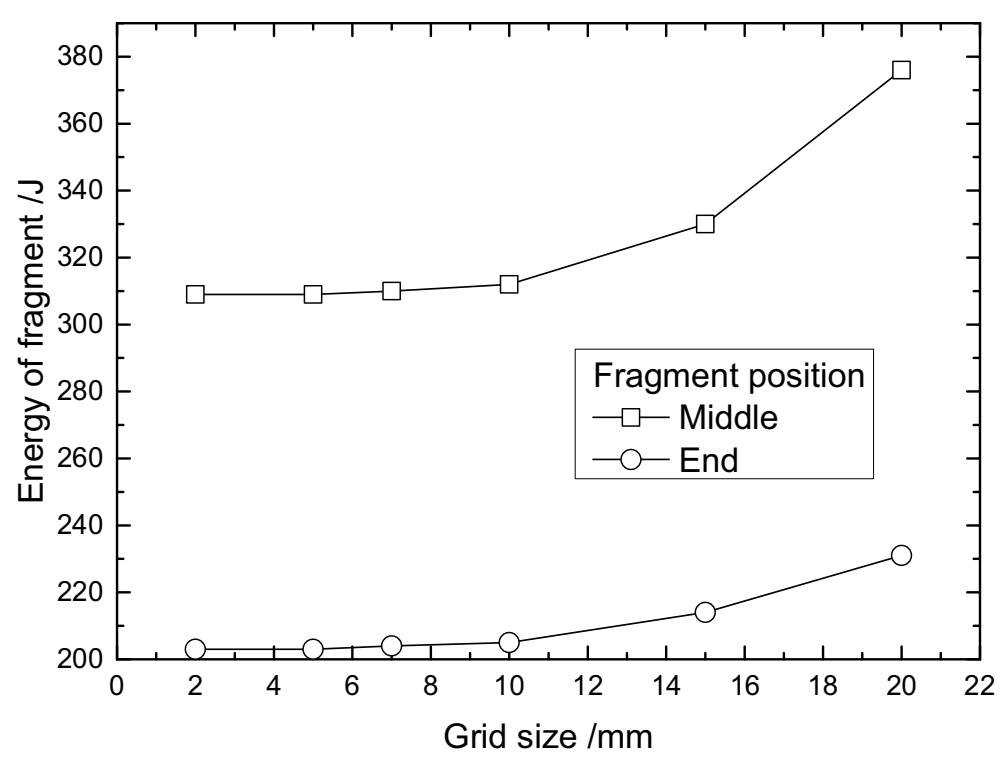

Fig 10. Fragments` energy for different discretizations.

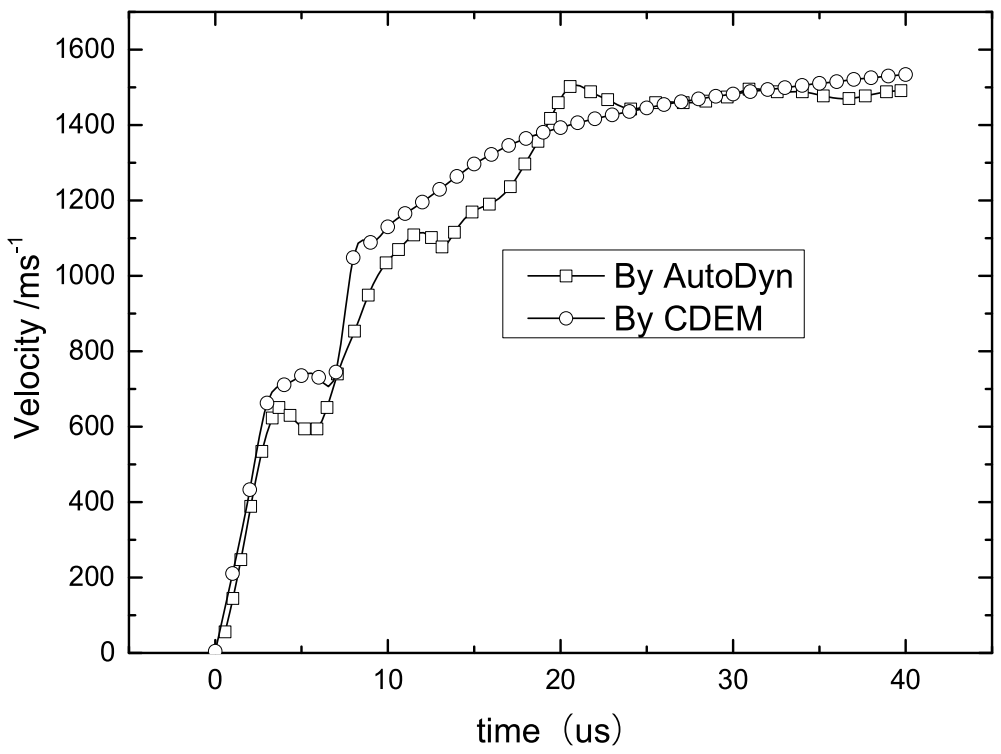

Fig 11. Fragment acceleration over time at the warhead body center.

Meanwhile, the shear and tensile strength are reduced based on current equivalent plastic shear and volumetric strain, as follows:

$\left.\begin{array}{l}\tau_{c}(t+\Delta t)=-\tau_{c 0} \times \gamma_{p} / \gamma_{\lim }+\tau_{c 0} \\ \sigma_{t}(t+\Delta t)=-\sigma_{t 0} \times \varepsilon_{p} / \varepsilon_{\lim }+\sigma_{t 0}\end{array}\right\}$

where $\tau_{c}(t+\Delta t)$ and $\sigma_{\mathrm{t}}(t+\Delta t)$ are the shear and tensile strength values in the next period, $\Delta t$ is the time step, $\tau_{\mathrm{c} 0}$ and $\sigma_{\mathrm{t} 0}$ are the initial shear and tensile strength values, $\gamma_{\mathrm{p}}$ and $\varepsilon_{\mathrm{p}}$ are the current equivalent plastic shear and volume strain, and $\gamma_{\lim }$ and $\varepsilon_{\lim }$ are shear fracture and tensile fracture strain.

The formula for calculating equivalent plastic volume strain is as follows:

$\varepsilon_{p}=\varepsilon_{x x}^{p}+\varepsilon_{y y}^{p}+\varepsilon_{z z}^{p}$

Plastic shear strain is calculated as follows:

$\gamma_{p}=\sqrt{\left(\varepsilon_{x x}^{p}-\varepsilon_{p} / 3\right)^{2}+\left(\varepsilon_{y y}^{p}-\varepsilon_{p} / 3\right)^{2}+\left(\varepsilon_{z z}^{p}-\varepsilon_{p} / 3\right)^{2}+2\left(\varepsilon_{x y}^{p}\right)^{2}+2\left(\varepsilon_{y z}^{p}\right)^{2}+2\left(\varepsilon_{x z}^{p}\right)^{2}}$

The value of input parameter of fragment equivalent layer is of great importance to numerical simulation. In actual performed fragment warhead, as shown in Fig 3, fragments are poured in resin around inner casing. Therefore, a single metal-resin casting could be considered as the minimum structure of fragment layer.

The fragment and resin in the structure expand together during practical detonation process, and the fracture and destruction will firstly occur in resin part obviously. Thus the determination of several equivalent layer parameters $\left(\mathrm{X}_{\mathrm{EF}}\right)$ in $\mathrm{CDEM}$ is summarized in Table 1.

\subsubsection{Finite element contact algorithms}

To detect contacts between polyhedrons efficiently and compute contact forces precisely, a combined semi-spring\&semi-edge contact model was proposed by our former study [29]. Here this combined contact model is used to calculate collisions between shell elements, shell elements and equivalent layer elements, and between explosive elements with a hole inside.

The semi-spring is formed by shrinking the vertices of each element into edges (two-dimensional) or surfaces (three dimensional); semiedges only act in three dimensions, and are formed through connections 


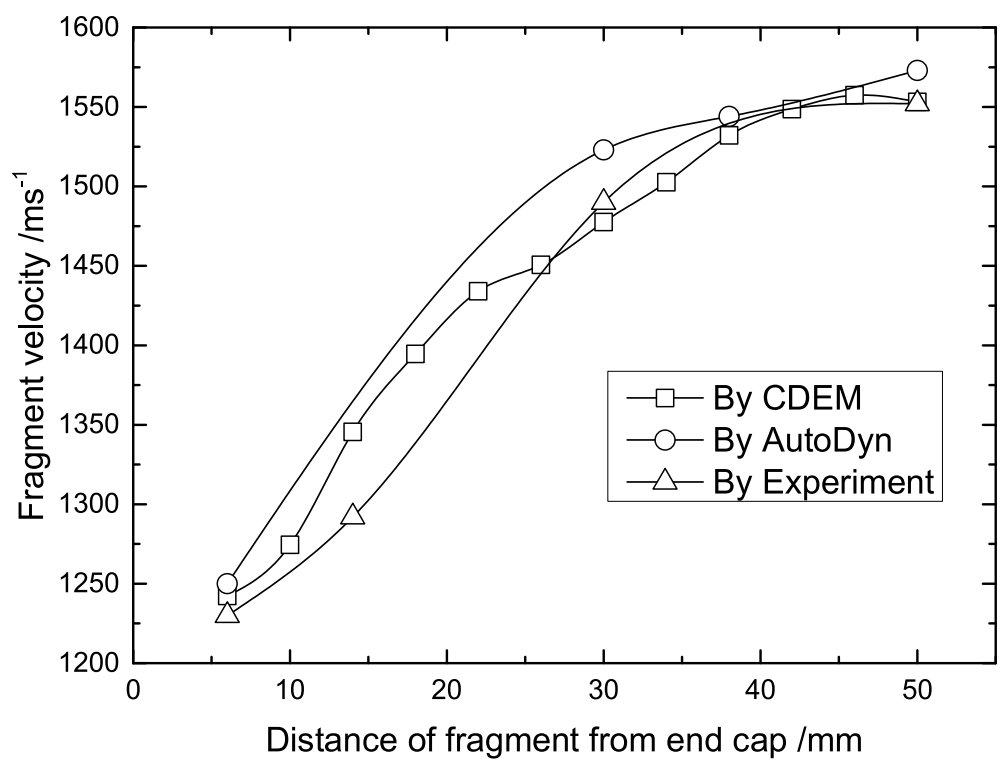

Fig 12. Distribution of fragment velocity over the warhead body.

Table 3

Classification of sensitivity.

\begin{tabular}{lll}
\hline & Index value & Classification \\
\hline 1 & $0.00 \leq \mathrm{I} \leq 0.05$ & Insensitive \\
2 & $0.05 \leq \mathrm{I} \leq 0.20$ & Commonly sensitive \\
3 & $0.20 \leq \mathrm{I} \leq 1.00$ & Sensitive \\
4 & $\mathrm{I} \geq 1.00$ & Very sensitive \\
\hline
\end{tabular}

between adjacent semi-springs in each surface (Fig. 4). The two-dimensional triangle in Fig. 4 includes six semi-springs, and the threedimensional tetrahedron contains 12 semi-springs and 12 semi-edges. When creating semi-springs, the shrinking distance is frequently $1 \%-5 \%$ (usually $5 \%$ ) from the vertex to the center of each edge or surface. The semi-spring and semi-edge only form complete contact pairs after finding their corresponding target surface or edge, hence the term "semi" (Fig. 5).

Since the semi-springs are located in each edge (two-dimensional), and the semi-edges are located in each surface (three dimensional), they each have their own characteristic area (unit thickness in two dimensions).

After the contact pairing is established, normal and tangential penalty springs are established on each pairing, and the elastic contact force is calculated from Formula 18, in which $F_{n}$ and $F_{s}$ are the normal and tangential contact forces, $K_{n}$ and $K_{s}$ are the normal and tangential contact stiffness, and $\Delta d_{n}$ and $\Delta d_{s}$ are the normal and tangential relative displacement increments.

$\left\{\begin{array}{l}F_{n}(t+\Delta t)=F_{n}(t)-K_{n} \times \Delta d_{n} \\ F_{s}(t+\Delta t)=F_{n}(t)-K_{s} \times \Delta d_{s}\end{array}\right.$

Formula 19 is used to correct the normal and tangential contact forces (tension is negative).

$\left\{\begin{array}{ccc}(1) \text { If } & -F_{n} \geq 0 & F_{n}=F_{S}=0 \\ (2) \text { If } & F_{S} \geq F_{n} \times \tan (\phi) & F_{S}=F_{n} \times \tan (\phi)\end{array}\right.$

\subsection{Particulate discrete element calculations}

\subsubsection{Fragment velocity input}

With the driven of the explosive pressure, the velocity of equivalent fragment firstly accelerated sharply and then reaches a constant stage gradually. Once the velocity reaches the constant stage, the velocities of its finite elements are mapped to the fragments (particles). If the particle's center of a given fragment is located within a given finite element, the following mapping formula can be used:

Table 4

Input parameters and simulation results.

\begin{tabular}{|c|c|c|c|c|c|c|}
\hline $\begin{array}{l}\text { Input } \\
\text { Parameter }\end{array}$ & Initial value & Variance ratio & $\begin{array}{l}\text { Output: KE of fragment } \\
\text { Middle of warhead }\end{array}$ & End of warhead & $\begin{array}{l}\text { Sensitivity index } \\
\text { Middle of warhead }\end{array}$ & End of warhead \\
\hline \multirow[t]{2}{*}{ Density of fragment layer $\left(\mathrm{kg} / \mathrm{m}^{3}\right)$} & \multirow[t]{2}{*}{5000} & $\uparrow 10 \%$ & $314.08 \mathrm{~J}$ & 206.18 & $0.153(0.19)$ & 0.151 \\
\hline & & $\downarrow 10 \%$ & $304.96 \mathrm{~J}$ & 200.27 & $0.142(0.2)$ & 0.14 \\
\hline \multirow[t]{2}{*}{ Tensile strength of fragment layer (Mpa) } & \multirow[t]{2}{*}{100} & $\uparrow 10 \%$ & 309.43 & 203.23 & 0.0026 & 0.0058 \\
\hline & & $\downarrow 10 \%$ & 309.26 & 202.99 & 0.0029 & 0.0059 \\
\hline \multirow[t]{2}{*}{ Young's modulus of fragment layer (Mpa) } & \multirow[t]{2}{*}{$1.05 \mathrm{e} 5$} & $\uparrow 10 \%$ & 309.44 & 203.17 & 0.0029 & 0.0031 \\
\hline & & $\downarrow 10 \%$ & 309.27 & 203.05 & 0.003 & 0.0034 \\
\hline \multirow[t]{2}{*}{ Poisson ratio of fragment layer } & \multirow[t]{2}{*}{0.32} & $\uparrow 10 \%$ & 309.4 & 203.14 & 0.0015 & 0.0014 \\
\hline & & $\downarrow 10 \%$ & 309.31 & 203.09 & 0.0011 & 0.0011 \\
\hline \multirow[t]{2}{*}{ Density of explosive $(\mathrm{kg} / \mathrm{m} 3)$} & \multirow[t]{2}{*}{1630} & $\uparrow 10 \%$ & 345.1 & 227.68 & $1.16(0.81)$ & 1.21 \\
\hline & & $\downarrow 10 \%$ & 257.8 & 170.68 & $1.20(0.92)$ & 1.19 \\
\hline \multirow[t]{2}{*}{ Detonation velocity of explosive $(\mathrm{m} / \mathrm{s})$} & \multirow[t]{2}{*}{6930} & $\uparrow 10 \%$ & 337.9 & 219.1 & $0.92(2.1)$ & 0.79 \\
\hline & & $\downarrow 10 \%$ & 281.2 & 187.9 & $0.91(2.1)$ & 0.75 \\
\hline
\end{tabular}

The theoretical results from Gurrny formula are enclosed in brackets.

Original value of fragments` energy in middle and end of the warhead body is $309.35 \mathrm{~J}$ and $203.11 \mathrm{~J}$ respectively. 


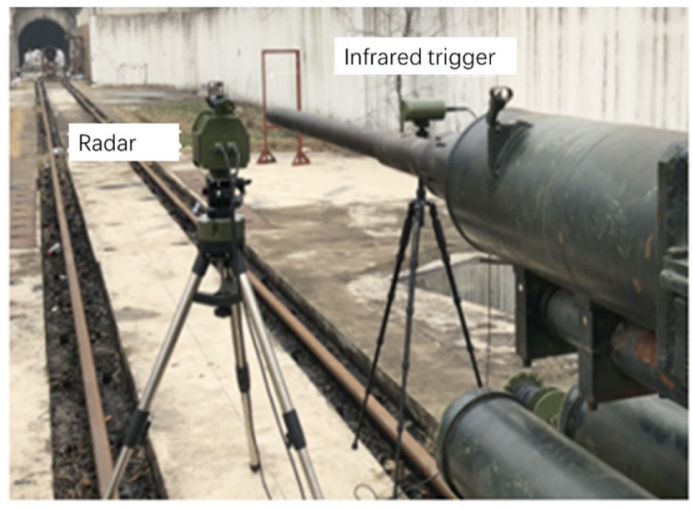

(a)

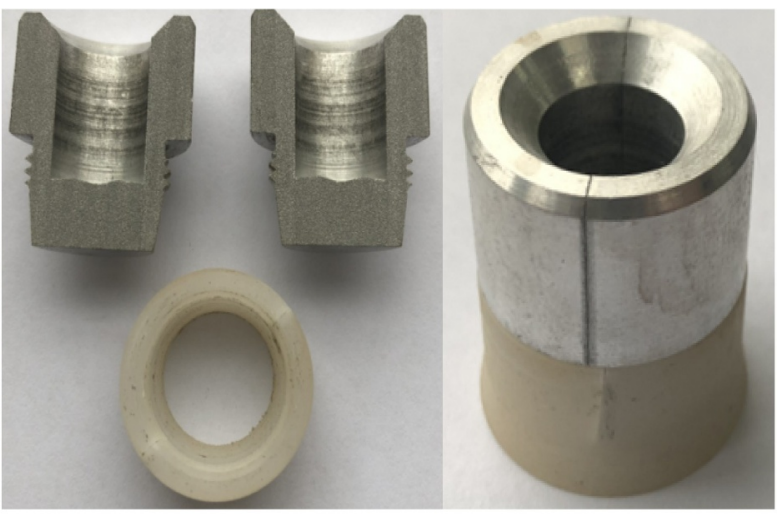

(b)

Fig 13. (a) Ballistic gun (b) Sabot.

Table 5

Test fragment parameters.

\begin{tabular}{llllll}
\hline No. & Material & Dimensions $(\mathrm{mm})$ & Windward area $\left(\mathrm{m}^{2}\right)$ & Mass $(\mathrm{g})$ & Initial velocity $(\mathrm{m} / \mathrm{s})$ \\
\hline 1 & 93 Tungsten alloy & $8 \times 8 \times 7$ cuboid & $9.16 \mathrm{e}-5$ & 7.9 & $1290-2000$ \\
2 & 93 Tungsten alloy & 98 sphere & $5.024 \mathrm{e}-5$ & 4.7 & $1340-1710$ \\
\hline
\end{tabular}

$\mathbf{v}_{i}^{\text {dem }}=\sum_{k=1}^{n} w_{k} \mathbf{v}_{k}^{\text {fem }}$

where $\mathbf{v}_{i}^{\text {dem }}$ is the velocity vector of a fragment, $\mathbf{v}_{k}^{\text {fem }}$ is the velocity vector of the $k^{\text {th }}$ node of a finite element, $w_{k}$ is the weight coefficient of the $k^{\text {th }}$ node, and $n$ is the number of nodes.

In Fig. 6, the velocity of fragment $i$ comes from that of finite element $j$.

\subsubsection{Fragment flight model with air resistance}

A particle flying model controlled by gravity force and drag force is suggested. Similar to interaction approach of FEM in Eq. (5), the forward-difference approximation is adopted to calculate flying process.

$\left\{\begin{array}{l}\mathbf{a}(t)=\mathbf{F}(t) / m_{p} \\ \mathbf{v}(t)=\mathbf{v}(t-\Delta t)+\mathbf{a}(t) \Delta t \\ \mathbf{u}(t)=\mathbf{u}(t-\Delta t)+\mathbf{v}(t) \Delta t\end{array}\right.$

where, $\mathrm{a}(\mathrm{t}), \mathrm{v}(\mathrm{t}), \mathrm{u}(\mathrm{t}), \mathrm{F}(\mathrm{t})$ are current acceleration vector, velocity vector, displacement vector and force vector, $\mathbf{v}(t-\Delta t)$ and $\mathbf{u}(t-\Delta t)$ are velocity vector and displacement vector at last step, $\mathrm{m}_{\mathrm{p}}$ is mass of fragment.

After velocity mapping, all fragments are assigned an initial velocity. The fragments decelerate in the air under the combined action of gravity and drag. The dynamics calculations in Formula 22, based on Newton's laws, to calculate the velocity and displacement of the fragments over time.

The resultant force on the fragments during flight is:

$\mathbf{F}=\mathbf{G}+\mathbf{F}_{c}$

where $\mathbf{F}$ is the resultant force vector, $\mathbf{G}$ is gravity force vector, and $\mathbf{F}_{c}$ is the drag force vector on the fragments during flight.

The drag $F_{c}$ is calculated as follows:

$\mathbf{F}_{c}=-\frac{1}{2} \xi \rho A|\mathbf{v}| \mathbf{v}$

where $\rho$ is air density (unit: $\mathrm{kg} / \mathrm{m}^{3}$ ), $A$ is the fragments' equivalent windward cross-section area (unit: $\mathrm{m}^{2}$ ), and $\xi$ is the coefficient of drag. In order to more accurately reflect the impact of drag on fragment flying velocity and trajectory, a velocity-dependent drag coefficient is used to solve for the drag $F_{c}$ (unit: $\mathrm{N}$ ). Based on previous research findings [30-32], for specific shape fragment, a correspondence relation exists between coefficient of drag and Mach number, as shown in Fig 7.

These curves in Fig. 7 are embedded in the calculation program, and the coefficient of drag force is calculated using the resultant fragment speed at a given moment, finally the drag force is obtained.

\subsection{Fragment field statistics}

In order to accurately calculate the long-range fragmentation effect, a penetrable, annular vertical virtual target plate of a certain height is proposed, centered on the warhead (Fig. 8).

If a fragment passes through a given target plate, the plate accumulates one more hit, and the velocity vector and position when it hits is recorded. A fragment's hit on the $i^{\text {th }}$ target plate is confirmed when it simultaneously meets both inequalities of Formula 24:

$\left\{\begin{array}{l}\left|R_{i}-r(t)\right|<t o l \\ z_{l} \leq z(t) \leq z_{u}\end{array}\right.$

where $R_{i}$ is the radius of the $i^{\text {th }}$ plate, $z_{l}$ and $z_{u}$ are the height of its lower and upper boundaries, $r(t)$ is the distance of a given fragment to the missile body at time $t, z(t)$ is the height of a given fragment at time $t$, and $t o l$ is the tolerance.

The numerical density of effective fragments hitting a given target $\rho_{d}$ can be calculated using Formula 25:

$\rho_{d}=\frac{N_{e}}{R_{i} H_{i} \theta}$

where $N_{e}$ is the number of effective fragments, $H_{i}$ is the height of the $i^{\text {th }}$ target, and $\theta$ is the scattering angle (radians).

\section{CDEM algorithm verification}

In this section, two tests are used to verify the accuracy of the CDEM calculations of the warhead fragment field over the full space-time domain for both close and long distances.

\subsection{Close-range verification}

AutoDyn is a widely used commercial program in the field of 


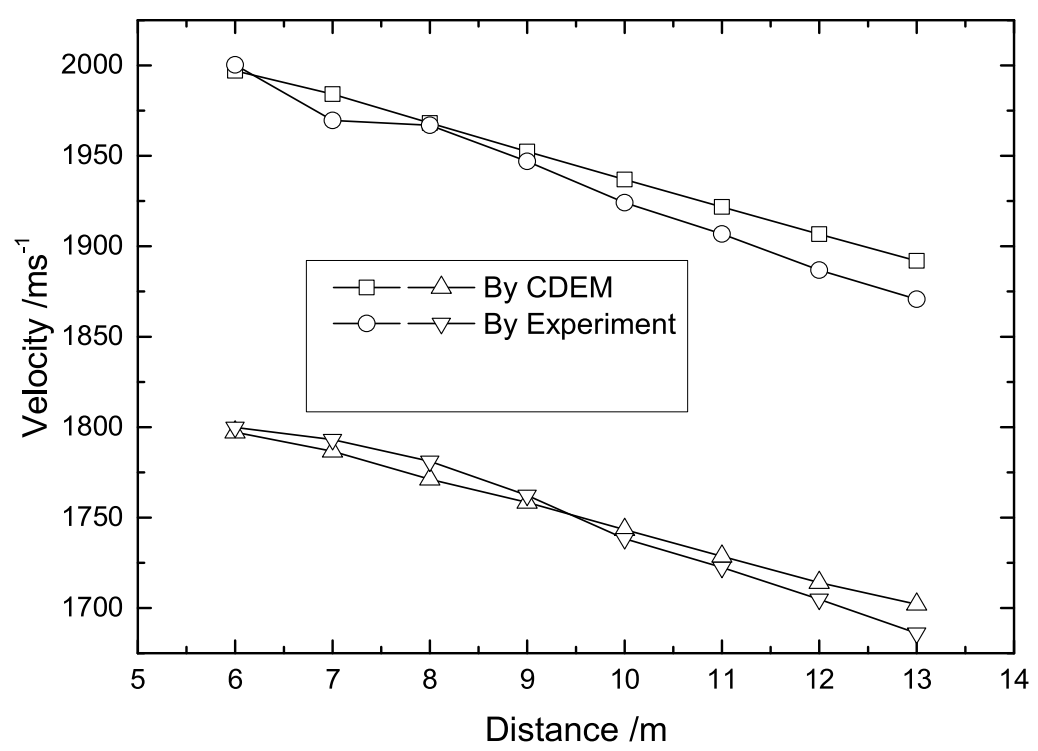

(a)

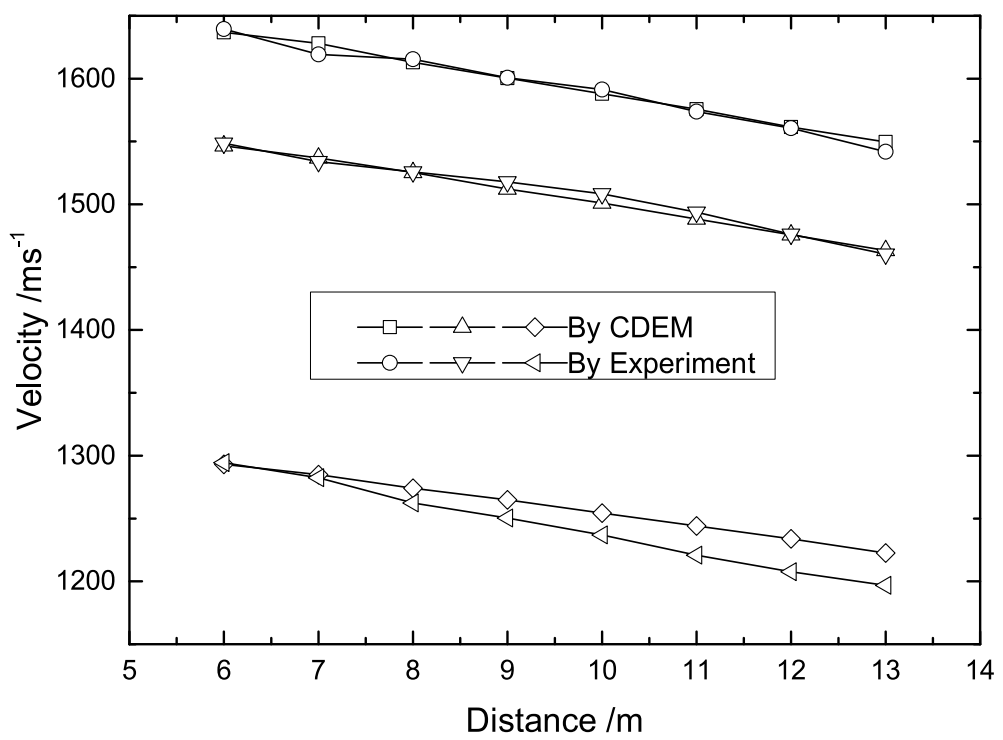

(b)

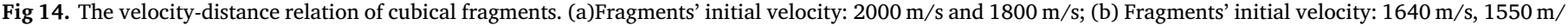
$\mathrm{s}$ and $1290 \mathrm{~m} / \mathrm{s}$.

warhead computing. Jiping et al. [33] used it to calculate the closerange fragmentation of a warhead and compare the calculations with experimental data. In order to verify the validity of the CDEM, trial calculations are conducted on the same warhead.

\subsubsection{Modeling}

The warhead is cylindrical, length $100 \mathrm{~mm}$, outer diameter $56 \mathrm{~mm}$. The detonation takes place at the center, as shown in Fig. 9. Some of the modeling parameters are as shown in Table 2:

\subsubsection{Grid independence analysis of CDEM}

In order to demonstrate the independence between computational results and discretizations, calculations with different size of the description grids $(20 \mathrm{~mm}, 15 \mathrm{~mm}, 10 \mathrm{~mm}, 7 \mathrm{~mm}, 5 \mathrm{~mm}, 2 \mathrm{~mm})$ are conducted respectively. Observation points are set on the middle and the end of the equivalent layer to collect kinetic energy of corresponding fragments.

As shown in Fig 10, the energy of fragments on the end and middle of warhead body both negatively affected by the size of grids. The calculation results remain generally stable when the grid size is less than $10 \mathrm{~mm}$. Thus, grids of $5 \mathrm{~mm}$ width(83.2e 3 grids in total) are applied in practical simulation, in consider of the calculation cost.

\subsubsection{Result of calculation}

The fragment acceleration curve at the center of warhead body and the distribution of initial fragment velocity over the warhead body are taken as examples for comparison.

It could be obtained from Figs. 11 and 12 that the CDEM fragment acceleration results over time from the center of the warhead body are close to those from traditional commercial finite element software; the CDEM and AUTODYN results for initial fragment velocity distribution fit well with experimental values. Notably, while the accuracy of both methods is comparable, because the former uses the equivalent fragment layer-detonation product escape algorithm rather than the fluidsolid coupling algorithm, its calculation time is much shorter, verifying the accuracy and efficiency of the finite element calculations in CDEM. 


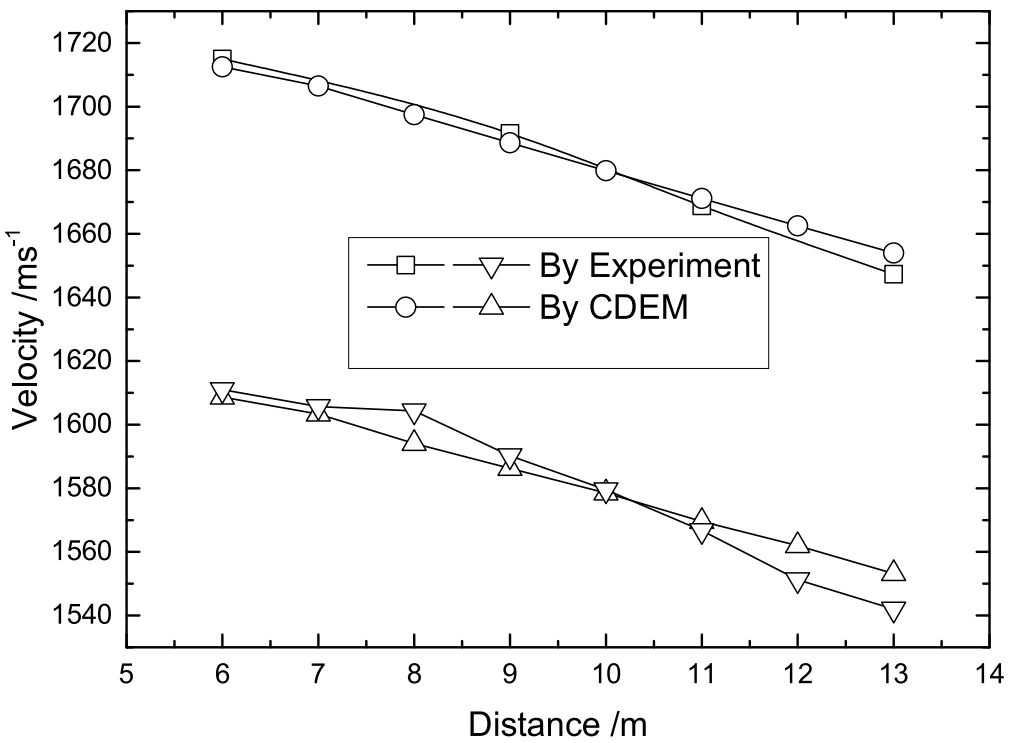

(a)

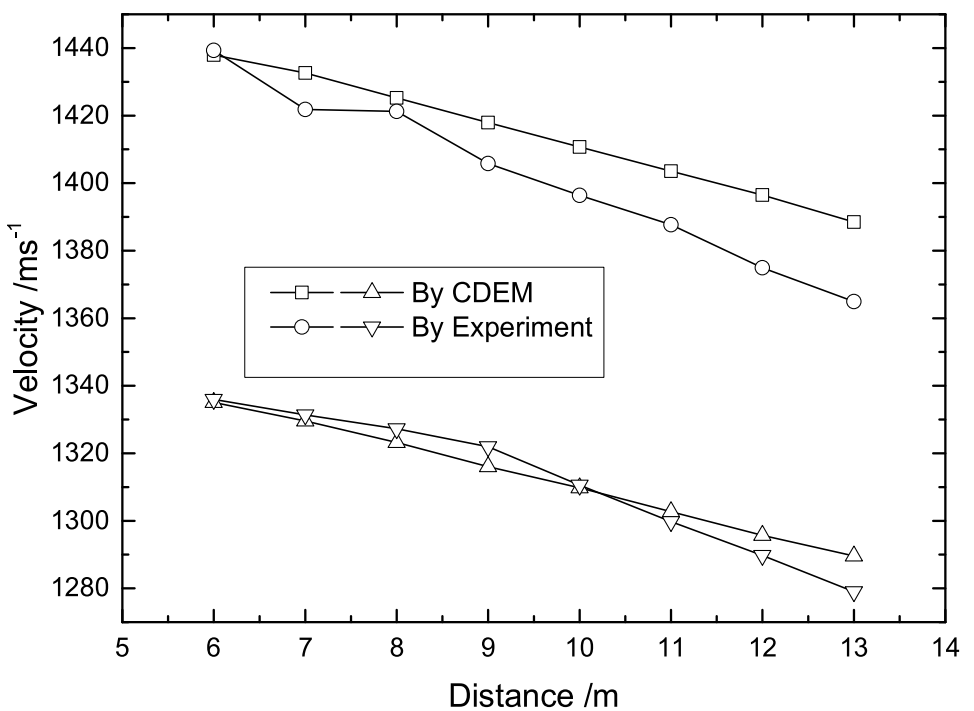

(b)

Fig 15. The velocity-distance relation of spherical fragments. (a)Fragments's initial velocity: $1710 \mathrm{~m} / \mathrm{s}$ and $1610 \mathrm{~m} / \mathrm{s}$; (b) Fragments's initial velocity: $1440 \mathrm{~m} / \mathrm{s}$ and $1340 \mathrm{~m} / \mathrm{s}$.

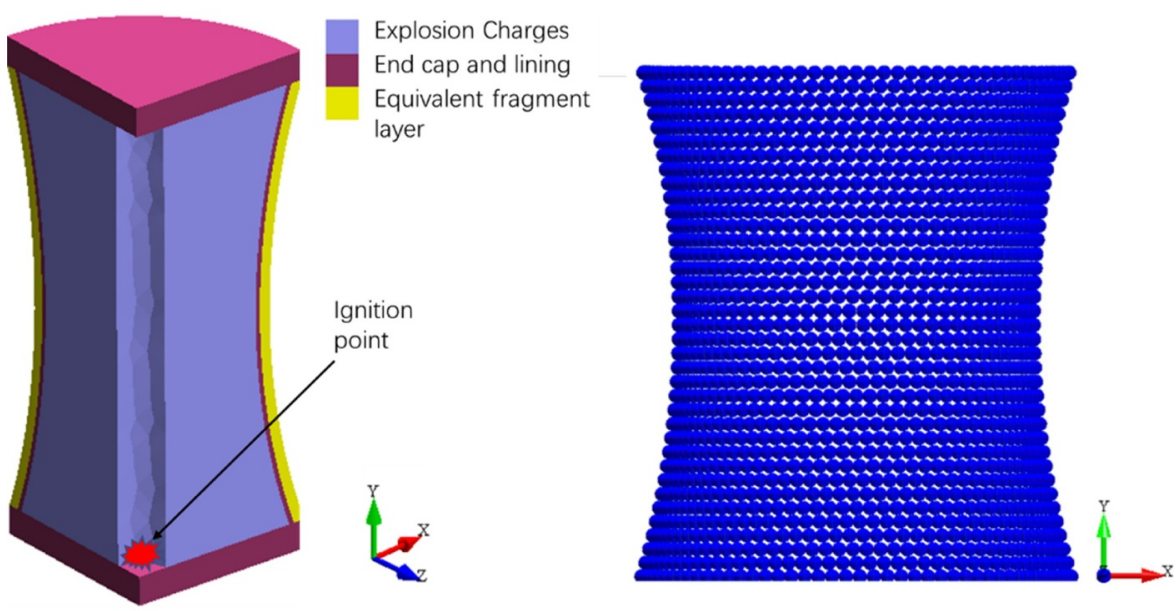

Fig. 16. Finite and particulate discrete elements modeling of warhead. 
Table 6

Warhead modeling material parameters.

\begin{tabular}{|c|c|c|c|c|c|c|}
\hline Location & Material & Density $\left(\mathrm{kg} / \mathrm{m}^{3}\right)$ & Tensile strength (Mpa) & Shear strength (Mpa) & Young's modulus (Mpa) & Poisson ratio \\
\hline End caps and inner casing & Aluminum alloy & 2700 & 410 & 410 & $0.71 \mathrm{e} 5$ & 0.3 \\
\hline Fragment equivalent layer & - & 7955 & 100 & 100 & $1.75 \mathrm{e} 5$ & 0.3 \\
\hline Fragments & Aluminum alloy & 15,200 & - & - & - & - \\
\hline
\end{tabular}

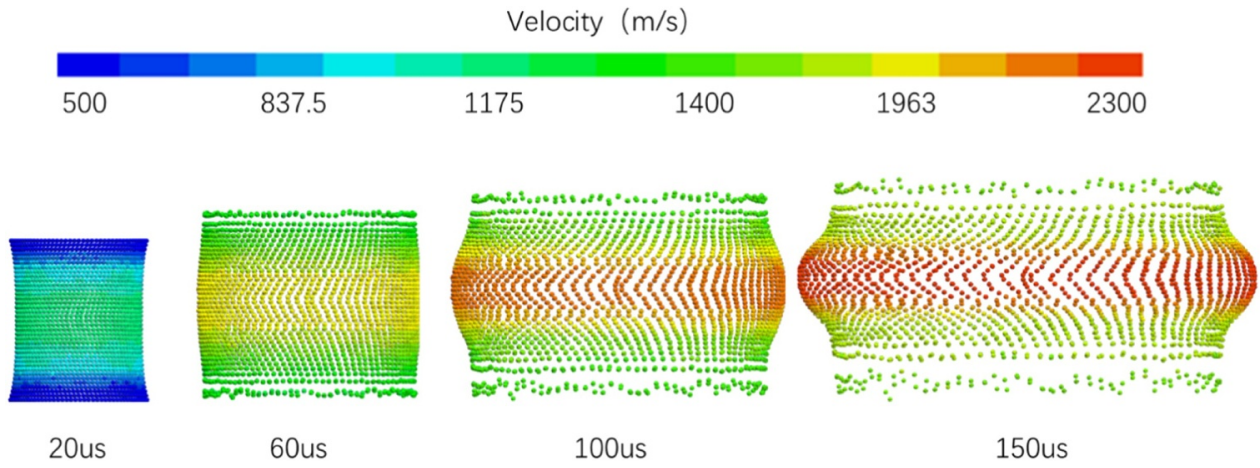

Fig. 17. Finite element calculations.

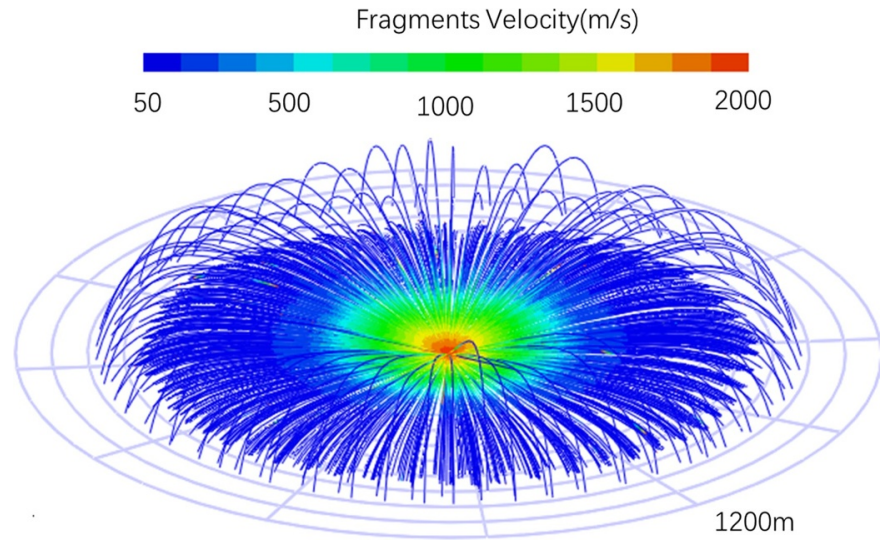

Fig 18. Fragment field.

\subsubsection{Sensitivity analysis of input parameters}

Finding the influential and non-influential parameters is of great significance to reducing complexity order of numerical problems, by which results in maintaining low computational costs. Therefore previous researchers, such as Morris [34], Khader et al. [14], have done a lot of sensitivity analysis(SA) studies on materials.

Variance-based methods is used in this paper. The variance based methods use a variance ratio to estimate the importance of input factors and primarily depend on the variance of the output response [14], which could be described using the Eq. (26):

$\mathrm{I}=\left(\Delta Y / \Delta F_{i}\right)\left(F_{i} / Y\right)$

where I is sensitivity index, by which the importance of input parameters could be classified, as is shown in Table $3 . Y$ is output result, $F_{i}$ is input parameters. $\Delta Y$ and $\Delta F_{i}$ are the variance of $Y$ and $F_{i}$ respectively.

For the sensitivity analysis of warhead calculation, the output of interest is the kinetic energy of fragments. The input parameters and calculation results are summarized in Table 4.

The density and detonation velocity of explosive charges are of the most great importance to the fragments' energy. The former one influences loading factor of warhead, and the later one affects explosive energy per unit mass, by which the total energy of warhead as well as the kinetic energy of fragments are changed.
The energy of fragments is commonly sensitive to the density of fragment`s layer. Heavier fragments layer expands more slowly, thus the processes of casing cracking and gas leakage is postponed. It means that more energy will be transferred to fragments when the total explosive energy is invariant.

The tensile strength, Young modulus and poisson ratio of fragment's layer have slightly influence on casing cracking and gas leakage, as well as the fragments` energy.

Gurrny formula (Eq. (27)) is widely used in calculating the fragment velocity of warhead theoretically [1].

$v=(D / 2) \sqrt{\frac{\beta}{2+\beta}}$

where $\mathrm{v}$ is the maximum velocity of fragments, $\mathrm{D}$ is the detonation velocity of charges. $\beta$ is the loading factor of warhead, defined as quality ratio of explosive to casing. The initial value of $\beta$ is 0.433 for this warhead.

Although, Gurrny formula has neglected some factors(such as the energy loss from shell deformation and gas leakage), the theoretical results of input parameter sensitivity classification fit well with computational results from CDEM.

The results of input parameter sensitivity classification from CDEM fit well with theoretical results from Gurrny formula. Some factors(such as the energy loss from shell deformation and gas leakage) has been neglected in Gurrny formula, result in deviation of numerical value of I from simulation.

\subsection{Experimental verification of fragment distance simulation}

Due to the large number of fragments in the warhead, their long flight distance, and complex trajectory, it is difficult to directly test the long-range warhead fragmentation field by experiment. The previous section has verified the accuracy of CDEM in calculating the short-range fragmentation field. The long range fragmentation field can be verified by verifying the simulation result of single fragment trajectory. Therefore, the research group loaded single fragment with high-speed ballistic gun system (Fig 13) and used millimeter-wave radar to track the velocity attenuation process continuously over long-distance flight for comparison with corresponding simulation results.

As shown in Table 5, two types of typical fragments are selected for 


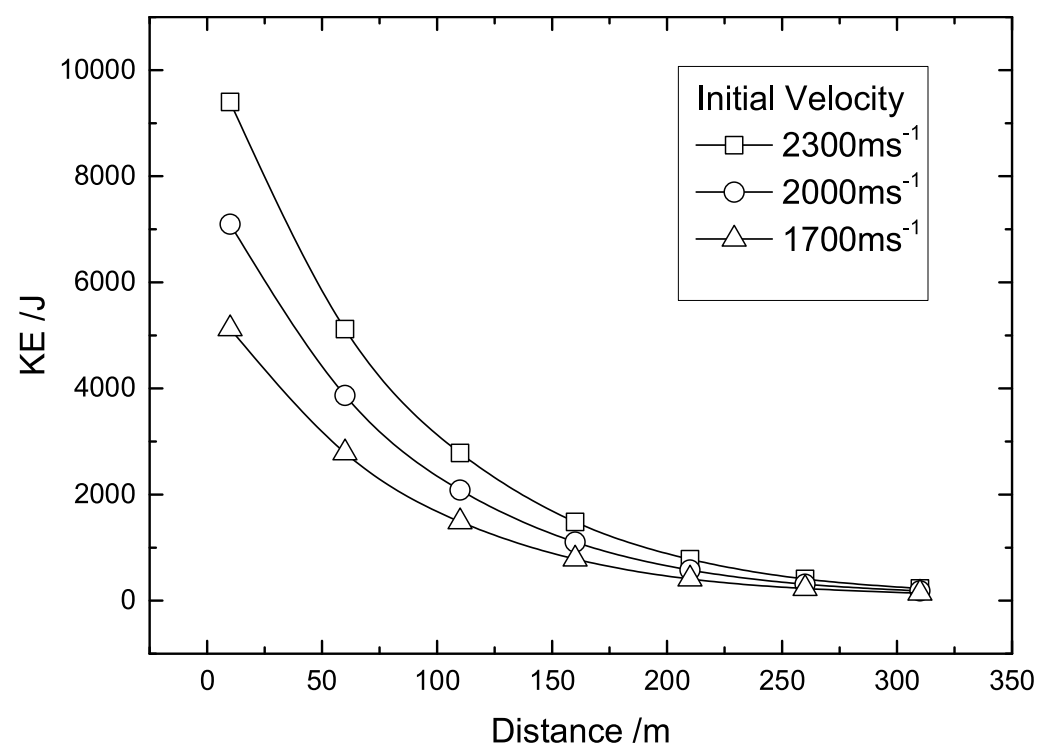

(a)

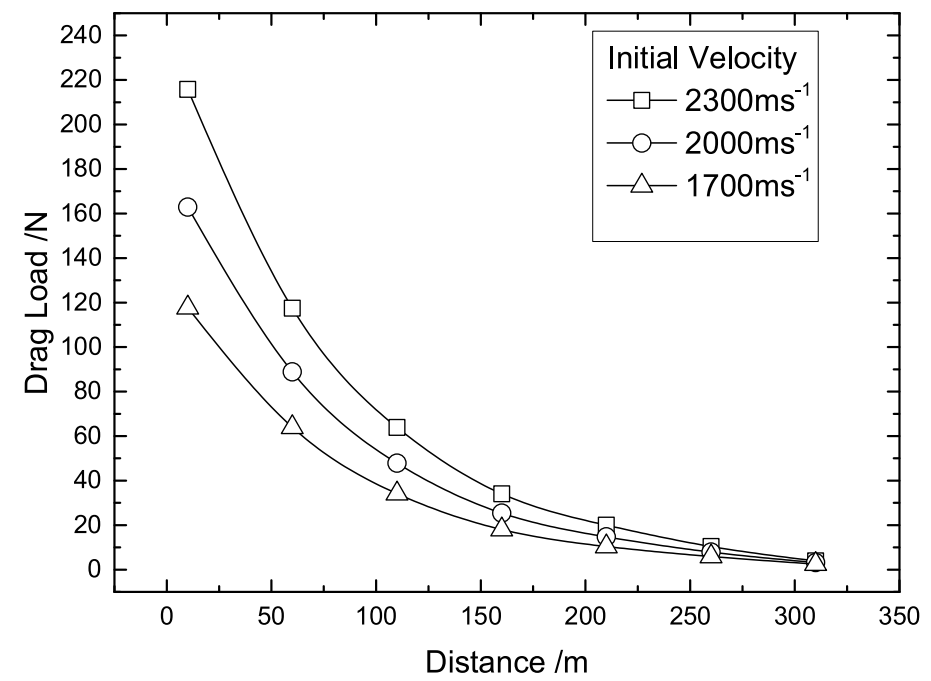

(b)

Fig 19. Variation of kinetic energy (a) and drag load (b) of fragments during flight.

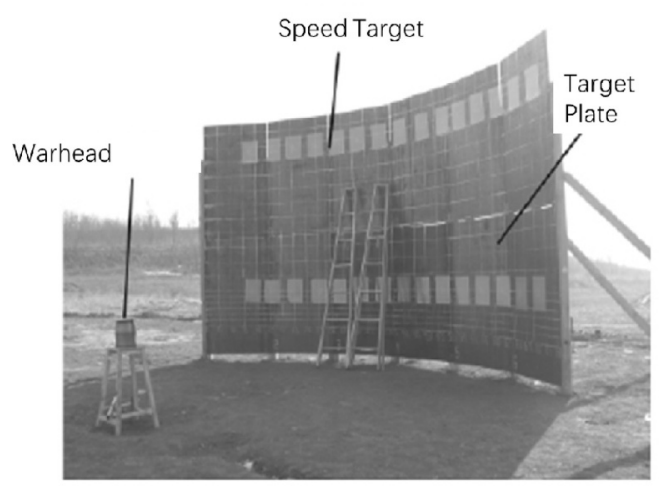

Target board

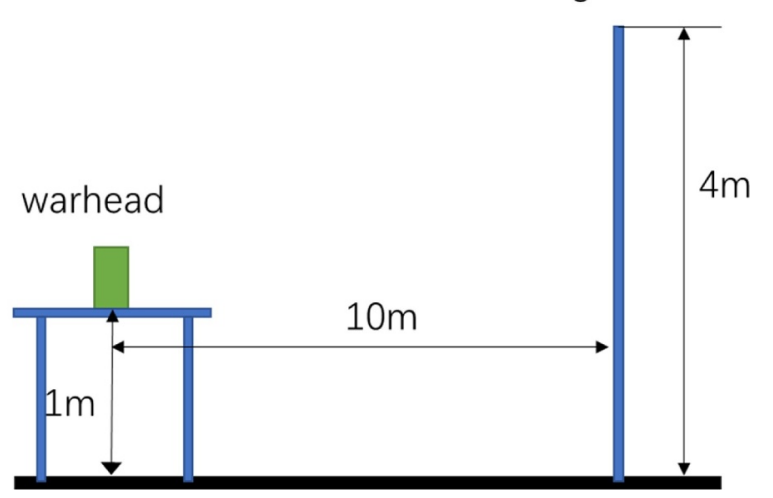

Fig 20. Static warhead explosion test layout. 


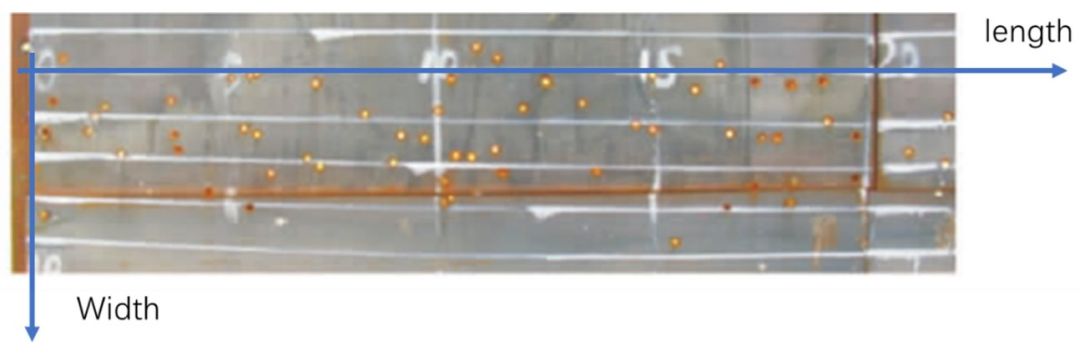

Fig. 21. photograph of target plate after experiment.

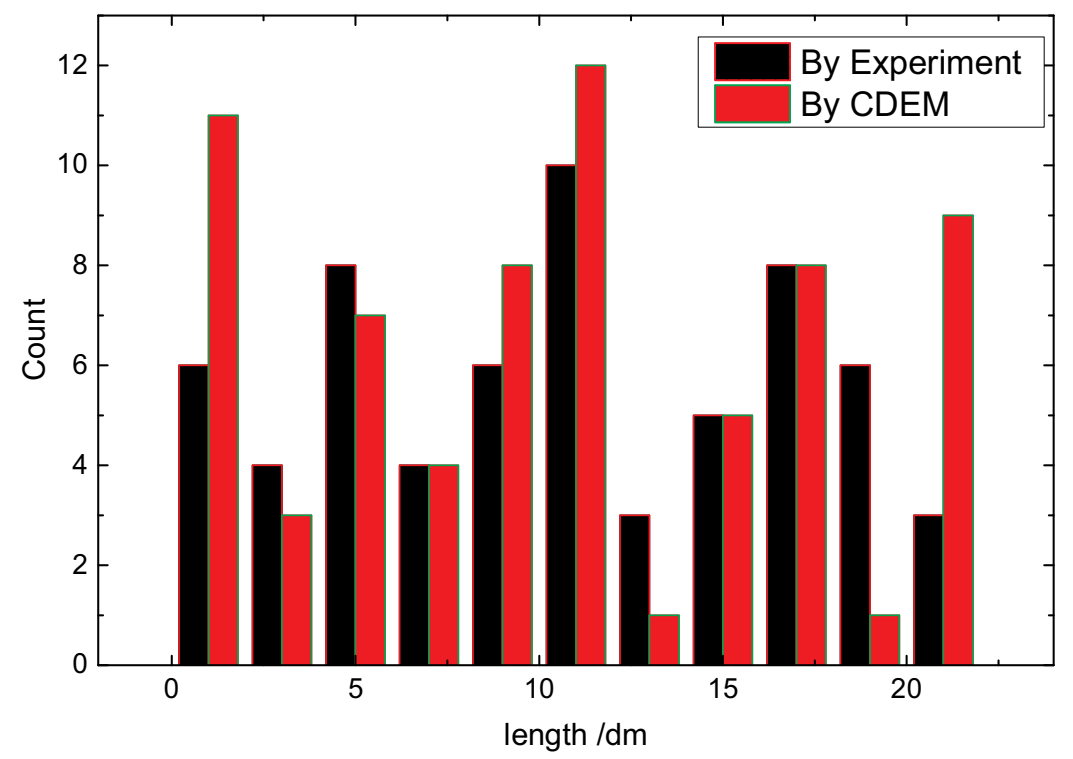

Fig. 22. Histogram of fragments on target plate(length direction).

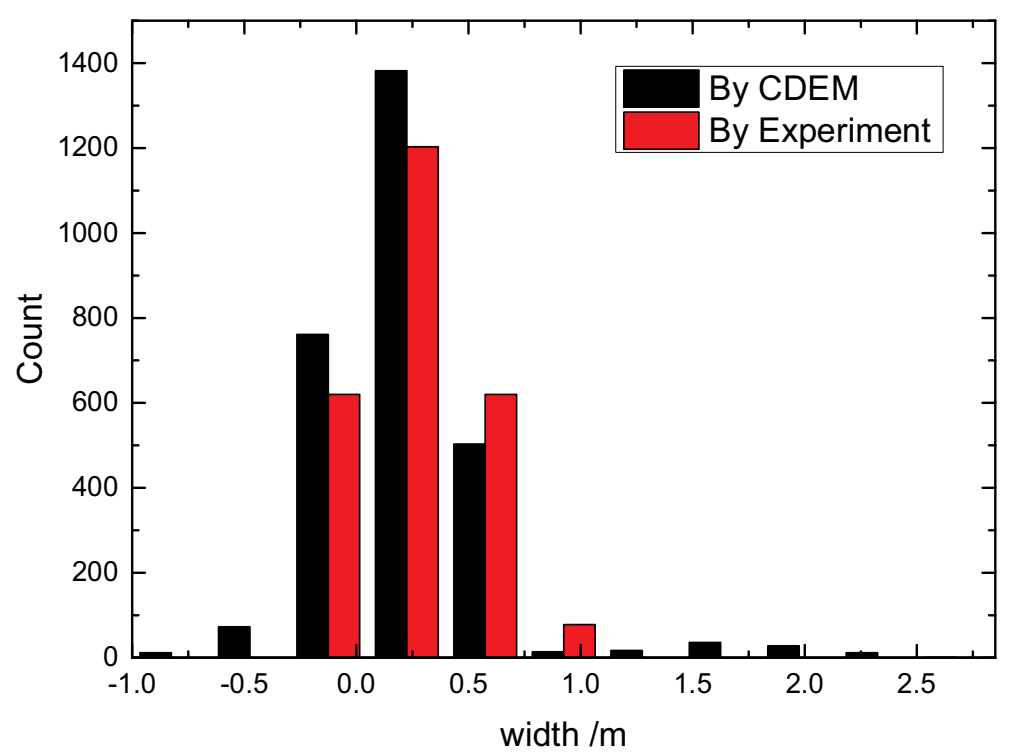

Fig 23. Histogram of fragments on target plate(Width direction).

testing to obtain the relationship between fragment velocity and flight distance.

The comparison with simulation results is shown in Figs. 14 and 15: The figures show that the maximum error between the simulation and experimental values is $2.4 \%$ (spherical fragments with initial velocity of $1440 \mathrm{~m} / \mathrm{s}$ at $13 \mathrm{~m}$ ), and the average error is less than $1 \%$, showing that CDEM accurately predicts long-distance fragment fields.

\section{Calculation and experimental verification of full fragment field over time and space}

Warhead modeling:

Consistent with Wang [35] , a typical focused warhead is selected for modeling, length $300 \mathrm{~mm}$, outer diameter $230 \mathrm{~mm}$, and concave in the middle of the body to focus the explosive energy. 2521 spherical 
Table 7

Comparison of simulation and test results.

\begin{tabular}{|c|c|c|c|}
\hline Item & Experimental value & Simulated value & Deviation \\
\hline Total fragment count in target plate & 70 & 68 & $2.94 \%$ \\
\hline Maximum fragment speed in target board $(\mathrm{m} / \mathrm{s})$ & 2253 & 2259 & $0.31 \%$ \\
\hline Selected focus band width (mm) & 350 & 350 & - \\
\hline Density of fragments in the focus band & 65.8 & 71.5 & $7.8 \%$ \\
\hline
\end{tabular}

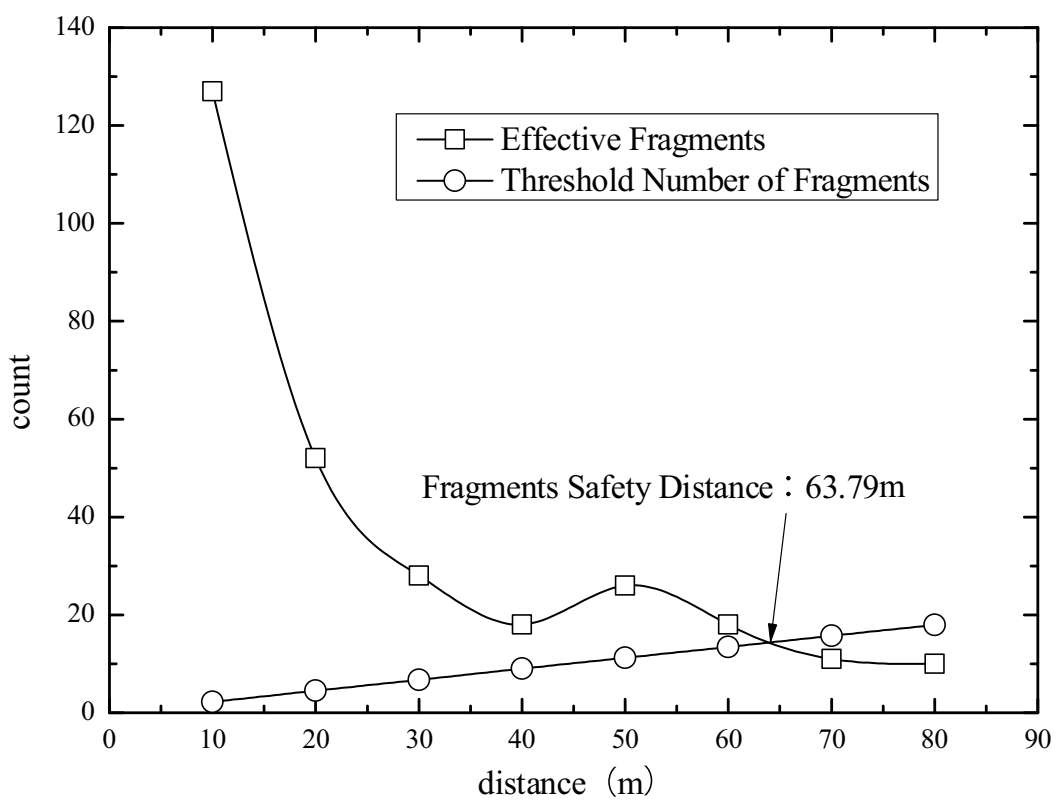

Fig 24. Fragmentation safety distance calculation.

tungsten alloy fragments weighing $4 \mathrm{~g}$ are set inside. The charge is HMX-based high-energy explosive, and the warhead is detonated from the center of the bottom end cap, as shown in Fig. 16.

For the finite element calculations, a Landau model is used for the explosives, with density $1780 \mathrm{~kg} / \mathrm{m}^{3}$, detonation speed $8070 \mathrm{~m} / \mathrm{s}$, and detonation pressure $32.6 \mathrm{e} 9 \mathrm{~Pa}$; an elasto-plastic model with strain softening is used for the aluminum inner casing, end covers, and fragment equivalent layer; and the tungsten fragments use a linear elastic model. The explosive, inner casing, end caps, and equivalent fragment layer are all described using Lagrangian grids of $10 \mathrm{~mm}$ width, 93,200 total number. The material parameters are as shown in Table 6:

For the particulate discrete element modeling, a dynamic air resistance coefficient which varies with velocity is used, and $1.069 \mathrm{~g} / \mathrm{L}$ is used for the air density (1000 $\mathrm{m}$ above sea level).

For target detection, the warhead is placed $1 \mathrm{~m}$ above the ground, and a $4 \mathrm{~m}$ tall annular virtual target plate is placed $10 \mathrm{~m}$ from the detonation center (the same size as the target plate in experiment).

Acceleration of casing pieces and fragments is calculated using finite elements, as shown in Fig. 17. After detonation, the warhead forms a clear focal zone in the center, with significantly higher fragment speed, consistent with the design of the warhead. Due to the bottom detonation, the highest initial velocity appears in the upper center of warhead body. With the escape of the detonation product, fragment acceleration decreases. After 150us, the fragment speed essentially reaches constant stage, at which point the maximum fragment velocity is $2286 \mathrm{~m} / \mathrm{s}$.

Long-range calculations then commence. The finite elements are deactivated and the discrete element module is activated to calculate the fragment flight. Due to the focusing effect, a large number of fragments in the focused beam have similar initial velocity and ejection elevation angle, so the fragmentation field extends outward over time in a ring configuration, as shown in Fig. 18.

The fragment field trajectory cloud chart shows that the fragment velocity decreases slowly, and the trajectory of each fragment remains relatively straight. The fragments are made of high-density tungsten alloy, each weighing $4 \mathrm{~g}$, giving them good speed retention and high ballistic coefficients.

As shown in Fig. 17, the fragments initial velocity distribution ranges from $1700 \mathrm{~m} / \mathrm{s}$ to $2300 \mathrm{~m} / \mathrm{s}$ approximately(150us). Several fragments with velocity in that interval are selected to obtain the reduction of energy and drag load during flight.

As shown in Fig 19, the kinetic energy and drag load of fragment are both negative exponentially attenuating with flight distance. Moreover, the attenuation rate is positively correlated with initial velocity of fragment. During long distances fight, the drag force of fragments will be balanced with gravity, and the velocity of fragments will be stable, which lead to the kinetic energy and load of fragments with different initial velocities tending to constant value eventually.

Static explosion tests of the same warhead were carried out, using velocity measurement targets and witness boards to collect the velocity and number density distributions of the fragment field. Static warhead explosion test layout is shown in Fig 20.

Fig 21 shows the photograph of target plate after experiment. Comparing the simulated fragment data on the vertical annular target board at $10 \mathrm{~m}$ with the test results, both are clearly focused in the width direction, the center height and the width of the focus zone from simulation coincide well with experiment. For the length, the fragments on the target plate also show a pattern of alternating density and sparsity, which is due to the limited number of radial prefabricated fragments in the warhead body, causing the horizontal flight angle of fragments to become discretized. The figure shows that the dense and sparse area of fragments on the target plate from simulation coincides with the experimental value, as shown in Fig. 22.

The above simulation results are processed into histogram with bins of $350 \mathrm{~mm}$, same as the experiment, to obtain the focus effect of 
warhead fragments as shown in Fig. 23:

The Table 7 compares the simulation results with the experimental values:

The fragmentation field data $10 \mathrm{~m}$ from the explosion center, using the CDEM described above, show good fit with the experiment, with no deviations over $10 \%$.

Using a $2 \mathrm{~m}$ permeable target board to collect long-range fragment statistics, the fragmentation safety distance is calculated using the methodology from DODs [36]:

After the warhead detonation, the fan-shaped azimuth range in which $90 \%$ of the fragments are concentrated is taken as the direction of danger.

Within the direction of danger, as the distance increases, when the fragments with kinetic energy greater than $78 \mathrm{~J}$ reach the density of $56 \mathrm{~m}^{2}$ /fragment, the distance is considered to be safety distance.

The Fig. 24 shows the relationship between effective fragmentation and distance. Its intersection with the threshold line ( $56 \mathrm{~m}^{2} /$ fragment) is the safety distance for the vertically placed warhead: $63.79 \mathrm{~m}$.

\section{Conclusions}

On the basis of the traditional continuous-discontinuous numerical simulation method, prefabricated fragment equivalent layer, detonation product escape, fragment air resistance coefficient association with Mach number, and penetrable vertical target board fragment collection algorithms are proposed, forming an analysis model which can fully simulate the fragment field in the full space-time domain, resolving the problems of large deformation and cross-scale calculation in warhead research. Comparison with experimental data verify the feasibility and reliability of this numerical analysis method.

The following conclusions can be drawn from the simulation results:

(1) CDEM is applicable for completely describing the whole process from breakage of the warhead casing to dispersion and landing of the fragments. No similar method has been found in current literature.

(2) The full-scale fragment field calculated by this method has high precision, and deviates less than $10 \%$ from experimental values.

(3) The fragment equivalent layer-detonation product leakage algorithm improves computing efficiency reducing the calculation time by more than $50 \%$ compared with commercial software, greatly saving resources.

(4) Combined with the penetrable target board system, the CDEM provides foundations to study fragmentation safety distance with different states and types of warhead in subsequent research.

In further research, the long-range testing (hundreds of meters) on actual warheads to directly verify the CDEM calculation results will be conducted. At the same time, based on this method, research on safety distance with different warhead configurations (focused, scattering, etc.), fragment types (tungsten alloy, steel, etc.), and initial warhead positions and movement status will be carried out.

\section{Data availability statement}

Data in this manuscript is good to offer, if there is any request, please contact Corresponding Author.

\section{Funding}

The research was funded by Major Special Projects of the National Defense Science and Technology Bureau. The associated grant number is not applicable due to confidentiality reasons.

\section{Declaration of Competing Interest}

The authors declare that there is no conflict of interest regarding the publication of this paper.

\section{Reference}

[1] Shuyuan Sui, Wang Shushan. Terminal effect. National defense Industry Pere; 2000.

[2] Powell JG, Smith WD, McCleskey F. Fragment hazard investigation program: natural communication detonation of $155 \mathrm{~mm}$ projectiles(ADA108775). Naval Surface Weapons Center; 1981.

[3] Marinko. Numerical simulation of the fragmentation process of high explosive projectiles. Sci Tech Rev 2013;63(2):47-57.

[4] Gold VM, Baker EL. A model for fracture of explosively driven metal shells. Eng Fract Mech 2008;75(2):275-89.

[5] Gold Vladimir M. Engineering model for design of explosive fragmentation munitions. USA: Armament Research, Development and Engineering Center; 2007. ADE403106.

[6] Hopson MV, Scott CM, Patel R. Computational comparisons of homogeneous and statistical descriptions of AerMet100 steel subjected to high strain rate loading. Int J Impact Eng 2011;38(6SI):451-5.

[7] Prytz AK, Odegardstuen G. Fragmentation of $155 \mathrm{~mm}$ artillery grenade, simulations and experiment. 26th International Symposium of Ballistics. 2011.

[8] Jian-Yu Chen, et al. Simulations for three-dimensional landmine detonation using the SPH method. J Impact Eng 2019;126:40-9.

[9] Glanville Jonathan P, Fairlie Greg, Hayhurst Colin. Numerical simulation of fragmentation using AUTODYNTM 2D \& 3D in explosive or dance safety assessment. 6th PARARI International Explosive Ordnance Symposium. 2003. p. 2-8.

[10] Rusinek A, Zaera R. Finite element simulation of steel ring fragmentation under radial expansion. Int J Impact Eng 2007;34(4):799-822.

[11] Zhang Zhi-chun, Qiang Hong-fu, Sun Xin-li. Numerical simulation for fragments flight characteristics of high explosive projectile. J PLA Univ Sci Technol (Nat Sci Edn) 2008;9(6):671-5.

[12] Li Weiping, Sun Hong, Zhang Haifeng. Numerical simulation of prefabricatedfragment warheads exploding based on ALE method. J Projectiles, Rockets, Missiles Guidance 2012;32(6):93-5.

[13] Hamdia Khader M, Silani Mohammad, et al. Stochastic analysis of the fracture toughness of polymeric nanoparticle composites using polynomial chaos expansions. Int J Fracture 2017;206(2):215-27.

[14] Hamdia Khader M, Ghasemi Hamid, et al. Sensitivity and uncertainty analysis for flexoelectric nanostructures. Comput Methods Appl Mech Eng 2018;337:95-109.

[15] Rabczuk Timon, Zi Goangseup, et al. A simple and robust three-dimensional cracking-particle method without enrichment. Comput Methods Appl Mech Eng 2010;199(37-40):2437-55.

[16] Rabczuk T, Belytschko T. A three-dimensional large deformation meshfree method for arbitrary evolving cracks. Comput Methods Appl Mech Eng 2007;196(29-30):2777-99.

[17] Shafiei A. Dynamic crack propagation in plates weakened by inclined cracks: an investigation based on peridynamics. Front Struct Civ Eng. 2018;12(4):527-35.

[18] Areias Pedro, Rabczuk Timon, et al. Element-wise fracture algorithm based on rotation of edges. Eng Fract Mech 2013;110:113-37.

[19] Oliver J. A consistent characteristic length for smeared cracking models. Int J Numer Methods Eng 1989;28(2):461-74.

[20] Gravouil A, Moes N, et al. Non-planar 3D crack growth by the extended finite element and level sets-Part II: level set update. Int J Numer Methods Eng 2002;53(11):2569-86.

[21] Wu Jian-Ying, Nguyen Vinh Phu. A length scale insensitive phase-field damage model for brittle fracture. J Mech Phys Solids 2018;119:20-42.

[22] W Haverdings. General description of the missile systems damage assecssment code (MISDAC). ADA288622, 1994.

[23] Moore Dedra, Reitmeier Paul, Deerman Brent. Safe separation modeling for aerial launched weapon systems. AIAA Modeling and Simulation Technologies Conference 08-11. 2011.

[24] Chrostowski JD, et al. HAZX Part1: an explosion hazard assessment tool. 2010 Department of Defense Explosives Safety Board Seminar. 2010.

[25] Wenshui Gan, Chrostowski, J.D.3D Fragment throw simulation to determine fragment density and impact on buildings. Department of Defense Explosives Safety Board Seminar (34th) held in Portland, Oregon.

[26] Jiang Jian-wei, Lu Yong-gang, Qian Li-xin. Application of shot-line model in simulation of fragment warhead. J Projectiles Rockets Missiles Guidance 2001;21(1):29-34. 10.15892 /j.cnki .djzdxb.2001.01.008.

[27] Chun Feng, Li Shihai, Liu Xiaoyu. Semi-spring contact model and its application to failure simulation of slope. Chin J Theoretical Appl Mech 2011:43(1):184-92.

[28] Chun Feng, Li Shihai, Zhang Bingxu. Numerical simulation on complete process of three-dimensional bench blasting in an open-pit mine based on CDEM. Explosion Shock Waves 2019;39(2). https://doi.org/10.11883/bzycj-2017-0393.

[29] Feng C, Li S, Liu X, et al. A semi-spring and semi-edge combined contact model in CDEM and its application to analysis of Jiweishan landslide. J Rock Mech Geotech Eng 2014;6(1):26-35.

[30] Moxnes John F, Brate Tom I, et al. Projected area and drag coefficient of high velocity irregular fragments that rotate or tumble. Defence Technol 2017;13:269-80.

[31] McCleskey F.Drag coefficients for irregular fragments. Naval surface warfare center (R15), NSWCTR 87-89.881227060. 1988 
[32] Miller M. Drag coefficient measurements for typical bomb and projectile fragments. Aberdeen Proving Ground, MD: August. US Army Research, Development and Engineering Center; 1990.

[33] Ji-ping Deng, Yi-ting Hu, Xian-zhen Jia. Numerical simulation of scattering characteristics of spherical fragment under Blasting. J Ballistics 2008;20(4):96-9.

[34] Morris MD. Factorial sampling plans for preliminary computational experiments. Technometrics 1991;33(2):161-74.

[35] Wang Juanjuan, Guo Shuangfeng. Numerical simulation and experimental study on directional-focused fragmentation warhead. Chin J Explosion Propellants 2013;36(2):87-90. https://doi.org/10.14077/j.issn.1007-7812.2013.02.017.
[36] MIL-STD-2105D HAZARD ASSESSMENT TESTS FOR NON-NUCLEAR MUNITIONS. U.S. 2011.

[37] Zhang Y, Zhuang X. Cracking elements: a self-propagating strong discontinuity embedded approach for quasi-brittle fracture. Finite Elem Anal Des 2018;144:84-100.

[38] Zhang Y, Lackner R, Zeiml M, Mang H. Strong discontinuity embedded approach with standard SOS formulation: Element formulation, energy-based crack-tracking strategy, and validations. Comput Methods Appl Mech Eng 2015;287:335-66.

[39] Zhang Y, Zhuang X. Cracking elements method for dynamic brittle fracture. Theo Appl Fract Mec 2019;102:1-9. 